\title{
Focused, Unfocused, and Defocused Information in Working Memory
}

\author{
Laura Rerko and Klaus Oberauer \\ University of Zürich
}

\begin{abstract}
The study investigated the effect of selection cues in working memory (WM) on the fate of not-selected contents of WM. Experiments 1A and 1B showed that focusing on 1 cued item in WM does not impair memory for the remaining items. The nonfocused items are maintained in WM even when this is not required by the task. Experiments 2 and 3 showed that items that were once focused in WM remain strengthened after the focus shifts away from them. When defocused items are presented as mismatching recognition probes, they are rejected better than other mismatching probes (Experiments 2 and 3). When a defocused item was later cued again, such that the focus had to shift back to it, that item was recognized better than an item cued for the first time (Experiment 3). The results support the distinction between mechanisms for temporary maintenance and the focus of attention in WM, and they challenge theories that explain maintenance and focusing by the same mechanisms, such as a limited number of slots or a limited resource.
\end{abstract}

Keywords: working memory, attention, focusing

Often, we have to hold several pieces of information in working memory (WM), but for a particular cognitive operation we need to focus on only a subset of them. What are the consequences of focusing for the remaining, unfocused information in WM, and what happens with the focused information once it is defocused later? In this article we investigate how focusing information affects nonfocused and defocused information in WM for visual information. We interpret the results within three theoretical frameworks for characterizing WM: theories assuming a discrete WM capacity (Cowan, 2005; Zhang \& Luck, 2008), constantresource theories (Bays \& Husain, 2008; Just \& Carpenter, 1992), and the three-embedded-components framework (Oberauer, 2002, 2009).

\section{Discrete-Capacity Theories}

Cowan (2001) introduced the hypothesis that WM has a discrete capacity of approximately four items or chunks. This capacity limit applies to the central component of Cowan's embedded-processes model of WM (Cowan, 1988, 1995, 1999). The model assumes two embedded structural components contributing to WM: the activated part of long-term memory (peripheral component) and the focus of attention (central component). The activated part of long-term memory facilitates retrieval of potentially relevant representations in long-term memory; it has no capacity limit but is limited by decay and interference. In contrast, the focus of atten-

This article was published Online First February 18, 2013.

Laura Rerko and Klaus Oberauer, Department of Psychology, University of Zürich, Zürich, Switzerland.

This research was funded by a grant from the Swiss National Science Foundation (project 100014_126766/1). We thank Matthias Hartmann, Iliana Karipidis, and Eszter Montvai for their assistance in the data collection.

Correspondence concerning this article should be addressed to Laura Rerko, Department of Psychology, University of Zürich, Binzmühlestrasse 14/22, 8050 Zürich, Switzerland. E-mail: 1.rerko@psychologie.uzh.ch tion is assumed to have a limited capacity, such that there is a maximum number of independent representational units (i.e., chunks) that can be maintained in the focus at any one time. ${ }^{1}$ This capacity limit is conceptualized as a discrete, fixed-capacity limit of about three to four units in healthy young adults (Cowan, 2001, 2005; Cowan, Rouder, Blume, \& Saults, 2012).

Zhang and Luck $(2008,2009)$ formalized the notion of a discrete capacity as a simple mathematical model for visual WM, called the slot model. The slot model proposes that chunks in WM are stored in an all-or-nothing fashion up to an individual limit, which is set by the number of available slots in WM. Only a single chunk (e.g., one object with all its features) can be stored in each slot. If the number of items to be stored exceeds the capacity limit, no more slots are available for storage and further items are lost to WM; consequently, participants can only guess about their identity, and retrieval of those items decreases to chance level.

Zhang and Luck (2008) proposed the slot-and-averaging model as a refinement of the slot model. According to the slot-andaveraging model, multiple copies of an item can be stored in multiple slots if more slots are available than there are items competing for them. Storing an item in more than one slot improves the precision with which that item's features can be retrieved, because information about this item from multiple slots is averaged, thereby reducing the variance of stored feature information. Assigning an item to multiple slots can also be used to prioritize that item; this provides a mechanism for focusing on an item within a set of items in the central component of WM. Because focusing within WM is the focus of our present work, we will use the slot-and-averaging model to derive predictions from discrete-capacity theories for our experiments.

\footnotetext{
${ }^{1}$ The capacity limit according to Cowan (2001) applies to chunks, which are learned units that can consist of several list items in an experiment. In the present context, each item of the memory set can be regarded as a separate chunk, and therefore we will from here on just speak of items or (in the case of visual WM) of objects.
} 


\section{Constant-Resource Theories}

Another conceptualization of the capacity limit in WM is to assume that a constant resource is responsible for the observed limit. Constant-resource theories have a long tradition in research on verbal WM (e.g., Just \& Carpenter, 1992); more recently, a constantresource theory has been proposed as an alternative to the slot model for visual WM (Bays \& Husain, 2008). The idea is that a constant limited resource can be flexibly distributed across the contents of $\mathrm{WM}^{2}$

By default, every representation can be assumed to receive an equal share of this resource (Bays \& Husain, 2008). With an increasing number of items to be stored, each item receives a smaller share of the resource, resulting in decreased accuracy of retrieval.

Because the resource can be assigned to representations flexibly, it is possible to focus on a subset of the representations in WM by assigning them a larger share of the resource. Importantly, the overall resource quantity remains constant. Therefore, if some representations are prioritized by receiving more of the resource, the resource apportioned to the remaining items must be decreased accordingly.

\section{The Three-Embedded-Components Theory of WM}

The three-embedded-components framework by Oberauer (2002, 2009) builds on the embedded-processes model (Cowan, 1995, 2001). The three embedded components can be understood as successive levels of selection of memory representations for (cognitive) action. The broadest level of selection is the activated part of long-term memory. It consists of the information that is potentially relevant for the task, similar to Cowan's (1999) conceptualization. The second component, the region of direct access, consists of a subset of representations in the activated part of long-term memory; these representations are temporarily bound to context cues through which they can be accessed. The region of direct access is similar to Cowan's focus of attention in that it is the central, capacity-limited component of WM. It differs from Cowan's theory, however, in that the capacity limit of the directaccess region is not a fixed number of chunks. Rather, the capacity limit arises from interference between item-context bindings. As the number of chunks to be remembered increases, chunks are not pushed out of the direct-access region but rather become increasingly difficult to discriminate (for details, see Oberauer, Lewandowsky, Farrell, Jarrold, \& Greaves, 2012).

Within the region of direct access, an individual item must often be selected as the target of the next cognitive operation. This item is held in the focus of attention, the narrowest level of selection in WM in the three-embedded-components framework. The focus is assumed to temporarily select a single item or chunk in the region of direct access. Note that the focus of attention in the theory of Oberauer $(2002,2009)$ differs from the focus of attention in the theory of Cowan (1999, 2005), in that it selects a single item within the set of items currently held in the central component of WM. The limitation of the focus of attention to a single item does not reflect a capacity limit but rather arises from its function as a selection mechanism: Selecting more than one item into the focus of attention is usually detrimental because these items cannot be kept apart, resulting in confusions and blends, unless the items in the focus are highly distinctive and participants are extensively trained (see Oberauer \& Bialkova, 2011; Oberauer \& Kliegl, 2004).
Evidence for the distinction between the region of direct access and activated long-term memory comes from studies showing that memory sets to which access is required for ongoing processing have a different status in WM than memory sets merely retained for a later test: Only the former produce set-size effects (Oberauer, 2001, 2005) and are reflected in ongoing neural activity (LewisPeacock, Drysdale, Oberauer, \& Postle, 2012). Evidence for the distinction between the region of direct access and the focus of attention comes from studies showing that, within a memory set held available for imminent access, the item most recently encoded or used has privileged status, as reviewed in the next paragraph. For a more detailed review of the evidence for the distinction of the three components, see Oberauer and Hein (2012).

In the context of recognition tasks, as in the present experiments, the distinction between the activated part of long-term memory and the direct-access region maps onto the distinction between familiarity and recollection in dual-process theories of recognition in WM (e.g., McElree \& Dosher, 1989; Oberauer, 2008). Familiarity is assumed to reflect whether a stimulus has been encountered recently without considering its bindings to contextual information. This kind of information is assumed to arise from activation of representations in long-term memory. In contrast, recollection yields information about the context in which an item has been encountered. Recollection draws on the bindings between items and their contexts in the region of direct access (Oberauer \& Lange, 2009). ${ }^{3}$

To prevent confusions due to the similar terminology in the models by Cowan (1995) and Oberauer (2002, 2009), we hereafter refer to Cowan's focus of attention and Oberauer's region of direct access by the theory-neural term central component of WM. The term focus of attention will be used for designating the narrow focus of attention in the three-embedded-components theory (Oberauer, 2002).

\section{Focusing Items Within Central WM}

There is converging evidence for the notion that people can prioritize individual items within central WM. Experiments involving sequential encoding or processing of information in WM have shown that the one item last encoded or last used is accessed particularly quickly and accurately (Garavan, 1998; McElree, 2001; Oberauer, 2006; Oberauer \& Bialkova, 2009; Woodman \& Vecera, 2011). For instance, repeatedly updating the same item

\footnotetext{
${ }^{2}$ Cowan et al. (2012) noted that the slot-and-averaging model introduced by Zhang and Luck (2008) takes an intermediate position between strict discrete-capacity theories (e.g., the slot model) and resource theories. They proposed further possible intermediate assumptions, such that a limited resource can be freely allocated up to a maximum number of elements. For the present experiments, these intermediate conceptualizations make the same predictions as the constant-resource hypothesis, and we therefore do not consider them separately.

${ }^{3}$ Familiarity of representations in activated long-term memory persists over several trials (Monsell, 1978) and thereby can create proactive interference from previous trials. Evidence for proactive interference in visual WM tasks such as ours is mixed (Lin \& Luck, 2012; Makovski \& Jiang, 2008; Shipstead \& Engle, 2012). In the present experiments, any proactive interference could not lead to systematic distortion of the results because the color arrays for each trial were created at random, such that there was no systematic relationship between the colors presented on the previous trials and the colors presented and tested in the current trial.
} 
leads to faster reaction times than does updating two different items in succession (Garavan, 1998; Oberauer, 2002). Likewise, repeatedly retrieving the same objects from WM to report different features of that object is easier than switching between objects (Woodman \& Vecera, 2011).

Several studies have investigated the beneficial effects of focusing attention in WM in response to a cue (e.g., Griffin \& Nobre, 2003; Landman, Spekreijse, \& Lamme, 2003; Makovski \& Jiang, 2007, 2008; Makovski, Sussman, \& Jiang, 2008; Nobre, Griffin, \& Rao, 2008; Sligte, Scholte, \& Lamme, 2008). Typically, variations of a visual short-term recognition paradigm (Luck \& Vogel, 1997) are used in those experiments. In these tasks, participants are required to memorize visual stimuli presented simultaneously in a memory display and to compare them (or parts of them) to a probe display. So-called retro-cues are presented in the interval between the offset of the memory display and the onset of the probe display, while the items are held in WM. These cues indicate which part of information from the memory display has to be compared to the probe display, thereby directing attention to specific WM contents. Using valid retro-cues leads to performance improvement compared to providing the same task with uninformative cues (Griffin \& Nobre, 2003; Lepsien, Griffin, Devlin, \& Nobre, 2005; Nobre et al., 2008) or with invalid cues (Griffin \& Nobre, 2003). This effect is referred to as the retro-cue benefit. Because the cue is presented after the memory display, the retro-cue benefit cannot be explained by a neglect of noncued items during encoding. Rather, it seems that attention can be applied to a single WM representation and that focusing on specific information in WM increases its accessibility.

In the experiments reported here, we used the retro-cue paradigm to induce people to focus a single object within a set of several visual objects held in central WM. We next consider how the three theoretical frameworks introduced above can be applied to the retro-cue benefit.

\section{Mechanisms of the Retro-Cue Benefit}

All three theories are compatible with the retro-cue benefit, but they differ in their predictions of the consequences of focusing on the nonfocused (noncued) representations within the central component of WM. According to the discrete-capacity theory, the retro-cue benefit can be explained by the assumption that the focused item is assigned to more than one slot, thereby gaining in precision through averaging (Zhang \& Luck, 2008, 2009). That is, instead of each object being assigned to a single slot, at least two slots are given to the focused object. As a consequence, however, fewer slots are available to the remaining, nonfocused information. Therefore, for memory displays exceeding the capacity, the fixed-capacity theory predicts that performance for noncued information suffers from focusing another representation. In other words, the retro-cue benefit implies a retro-cue cost for nonfocused information.

The constant-resource framework assumes that a limited resource can be flexibly allocated to single objects held in the central component of WM. According to this theory, the cuing benefit can be explained by assuming that a larger resource quantity is allocated to the cued item than to the other items (Bays, Gorgoraptis, Wee, Marshall, \& Husain, 2011). Thereby, its likelihood of being correctly retrieved is increased compared to that of the other items. Crucially, when the amount of resource for the focused item is increased, less of the resource is available for the remaining items in central WM. Hence, the retro-cue benefit again implies a cost for nonfocused information in the central component of WM, resulting in the same prediction for nonfocused information as in the fixed-capacity theory.

In the three-embedded-components theory (Oberauer, 2002, 2009), the focus of attention is separate from the capacity-limited central component of WM (i.e., the direct-access region). According to this theory, the retro-cue benefit arises because the cued object becomes the content of the focus of attention. Selecting an object into the focus enhances its accessibility but does not impair the representations of other objects in the direct-access region. Therefore, in contrast to the other two theories, no detrimental effect of the retro-cuing benefit is predicted for the nonfocused representations.

In sum, focusing information in WM prioritizes the focused item and causes a performance benefit if this information is required for the next cognitive action. The models do not differ regarding their predictions for the focused item, but they propose different fates for the remaining WM contents. The present study asks what happens to them. We distinguish nonfocused information (information that has not been prioritized during the retention interval) and defocused information (information that had been recently prioritized but then replaced by other information to be prioritized). Examining the fates of these kinds of information will provide information for evaluating the models introduced above. In the following section we detail the hypotheses we test in the present experiments and summarize the scant available evidence on the effects of focusing on the fate of nonfocused and defocused information.

\section{Nonfocused and Defocused Information in WM}

\section{Nonfocused WM Contents}

Nonfocused information refers to information inside central WM that has not been focused (attended) after initial encoding. ${ }^{4}$ As discussed above, fixed-capacity theories and constant-resource theories of WM lead to the prediction that, as one item is focused, other items in central WM tend to be forgotten more, compared to a situation where no item is focused. In contrast, the three-embeddedcomponents theory predicts that focusing one item has no effect on memory accuracy for the remaining items.

Support for increased forgetting of nonfocused information in WM was provided by a series of experiments by Matsukura, Luck, and Vecera (2007). They compared two different accounts for the beneficial effect of focusing in WM: the protection account and the prioritization account. The protection account assumes that focused attention protects the cued item from degradation and interference, while the nonfocused items decay or suffer from interference. In contrast, the prioritization account presumes that the cued

\footnotetext{
${ }^{4}$ Presumably, some kind of attention is needed to encoded stimuli into WM, but there are multiple forms of attention (Chun, Golomb, \& TurkBrowne, 2011), and it is not clear whether the focus of attention for selecting items within WM is involved in encoding items into WM. At present it is not clear whether encoding of simultaneously presented items occurs sequentially or in parallel (Bays et al., 2011; Jolicoeur \& Dell'Acqua, 1998). If encoding is sequential, the focus of attention could encode them one by one. However, if encoding is parallel, the single-item focus of attention cannot be involved in encoding of all stimuli. Whether or not the focus of attention is involved in encoding does not affect our conclusions.
} 
item is given priority in the comparison process, while noncued items remain available for later comparison. Matsukara et al. presented retro-cues pointing to either the left or the right hemisphere of the display in a visual WM paradigm. In a subset of trials, two successive cues were presented; the last of them was always valid. Two conditions were distinguished for the two-cue trials: Either both cues were pointing to the same hemisphere (e.g., set of items on one side of the fixation cross) or the two cues pointed in opposing directions. When both cues pointed in the same direction, memory performance was as good as in single-cue trials. When the second cue pointed into another direction than the first cue, performance was worse than in single-cue trials. Matsukara et al. interpreted this as support for the protection account, assuming that while participants focused on the hemisphere cued by the first cue, the information in the other hemisphere became prone to forgetting. This forgetting could be due to resource quantities or slots being taken away from the nonfocused items, but it could also be due to decay or interference acting on the nonfocused items during the time of the second cue. According to the latter interpretation, which was endorsed by Matsukura et al. (2007), it is not the act of focusing itself that impairs memory for the nonfocused information. Therefore, their result does not adjudicate between the three theories considered here.

Evidence for unimpaired maintenance of nonfocused information was provided by a study by Landman et al. (2003) using a change detection task. Change detection is a variant of the shortterm recognition paradigm in which participants compare a memory display to a probe display and determine whether they match or whether there was a change from the first to the second display. Landman et al. presented either one or two successive cues in the retention interval, indicating which single item from the memory display might change from memory display to probe. In the case of two cues, the last cue (which pointed in a direction other than the first cue) was always valid in predicting the location of the possible change. Additionally, onset time of the valid cue was manipulated (early vs. late). Results revealed no difference between early and late valid cue onset, and memory performance was not impaired by the first cue in two-cue trials compared to single-cue trials. These results indicate undiminished maintenance of nonfocused objects and flexible reallocation of attention, supporting the assumption that nonfocused information is retained in memory. Landman et al. (2003) presented the memory display and the two cues in rapid succession, so that it is not clear to what extent sensory memory contributes to performance.

So far, we have considered two possible fates of nonfocused information in WM, maintenance and forgetting. A third possibility is that nonfocused items are actively removed from central WM when the person is confident that the nonfocused information is no longer needed. An assumption of the three-embedded-components model is that information no longer needed can be removed from central WM, thereby reducing the interference that limits the capacity of central WM (Oberauer, 2002, 2005). Evidence for removal of information comes from experiments using a modified Sternberg task. In these experiments, two memory lists were presented for encoding, and a retro-cue indicated which of the two lists was relevant for a recognition test. Reaction times (RTs) for responses to the recognition probe revealed robust set-size effects for the relevant list, whereas the set-size effect of the irrelevant list disappeared with increasing time between the cue and the test stimulus. One to two seconds after the cue, the irrelevant set size had no influence on RTs. This time reflects the time it takes to remove nonfocused information from the central component of WM.

Active removal can be distinguished from passive forgetting because the former, but not the latter, is under flexible control, depending on task demands. Passive forgetting would affect nonfocused information regardless of its relevance, whereas a person would actively remove information only if it is perceived as no longer relevant. In Experiments $1 \mathrm{~A}$ and $1 \mathrm{~B}$, we tested whether people can flexibly control the fate of all noncued items by manipulating whether nonfocused information is rendered irrelevant or remains relevant.

\section{Defocused WM Contents}

The status of nonfocused information might differ from the status of previously focused and then defocused information. Both types of information are in central WM and currently not focused, but they differ regarding their focusing history. Nonfocused information has never been focused after encoding. In contrast, defocused information has recently been focused but is currently not focused anymore. Little research has been done on the status of defocused WM contents, and their fate has not been considered in the three WM theories introduced above. We therefore cannot derive predictions for defocused information from these theories. Nevertheless, three broad hypotheses regarding its fate can be contrasted: Defocused information can be set back to baseline level of memory strength, it can be inhibited, or it can be strengthened compared to nonfocused WM representations. In the context of Experiment 2 we will elaborate these hypotheses, and in the General Discussion we will discuss how our results can be accommodated by the three theories.

The present study investigates the fate of nonfocused and defocused information in WM for visual information. Experiments $1 \mathrm{~A}$ and $1 \mathrm{~B}$ provide support for the maintenance of nonfocused information in $\mathrm{WM}$, even if this information is not required for the task. Experiments 2 and 3 investigate the fate of defocused information and show that defocusing leaves the respective item strengthened relative to information that was not focused during the retention interval.

\section{Experiment 1A and 1B: Retro-Cue Benefit and Nonfocused Information in WM}

Our main aim in both Experiment 1A and Experiment 1B was to examine how prioritizing one item in WM affects memory for the remaining items. Furthermore, in Experiment 1A, we intended to replicate the retro-cue benefit in our task; that is, the finding that cues orienting attention to one item in WM improve retrieval accuracy for that item. For these purposes, we used a short-term color recognition task, which required participants to memorize a multi-item memory display and compare one of these items to a single-item probe display. The experimental design was modeled after the third experiment described in Landman et al. (2003): After the memory display was encoded, either one or two successive cues were presented. Participants were correctly informed that the last cue was always valid. Our experiments differed from Landman et al.'s (2003) experiment in three regards: First, we increased several time intervals to examine focusing effects on nonfocused information in WM-over a time course that unam- 
biguously excludes contributions from iconic memory. Second, in Experiment 1A, we added two no-cue conditions, serving as baselines to evaluate whether the retro-cues were beneficial to memory performance. Third, we examined whether the fate of nonfocused WM representations is under flexible control by manipulating the predictability of the number of retro-cues.

In line with the well-established retro-cuing benefit (e.g., Griffin \& Nobre, 2003; Lepsien et al., 2005; Nobre et al., 2008), cue trials were expected to show better performance than no-cue trials in Experiment 1A. To test whether noncued information remains accessible after attention was cued to another location, we compared performance in single-cue trials and two-cue trials. If noncued items are maintained in central WM and people can flexibly shift the focus of attention within it, performance on two-cue trials should be as good as that in singlecue trials with the same retention interval. In contrast, if focusing one item is detrimental to memory for the remaining nonfocused items, performance in single-cue trials should be better than performance in two-cue trials.

To assess whether people have control over whether they maintain or remove noncued information, we created two predictability conditions. In the predictable condition there were no two-cue trials, so that after seeing the first cue, participants knew that this cue validly pointed to the item that would be tested. Therefore, participants could remove nonfocused information from the central component of WM after receiving a cue, thereby reducing memory load and improving task performance. In the unpredictable condition, single-cue and two-cue trials (and no-cue trials in Experiment 1A) were mixed randomly, so that after seeing the first cue, participants did not know whether this cue would be valid in the end. In this condition, it would be unwise to remove all noncued items upon seeing the first cue, because doing so would jeopardize performance in two-cue trials. Therefore, in the unpredictable condition participants should rather maintain nonfocused information after the first cue. If participants followed their optimal strategy under each predictability condition, this would result in higher WM load in the unpredictable condition than in the predictable condition. Hence, comparing performance on single-cue trials between predictable and unpredictable conditions reveals whether nonfocused information can be flexibly maintained or removed according to task requirements.

To summarize, we can distinguish three possible outcomes: If nonfocused information is maintained in WM, we should observe no difference between single- and two-cue trials, as well as nondistinguishable performance between predictability conditions. In case nonfocused information is weakened or entirely lost, performance in single-cue trials should be better than in two-cue trials, regardless of predictability. Finally, in case of flexible control, performance in the predictable condition should be better than in the unpredictable condition on single-cue trials, and single-cue and two-cue trials should yield equal performance in the unpredictable condition. A summary of predictions can be found in Table 1.

Many studies investigating WM with visual materials asked participants to engage in articulatory suppression to ensure that they relied exclusively on visual, as opposed to verbal, WM. In this and the following experiments we did not use articulatory suppression for two reasons. First, we are interested in general mechanisms of WM, rather than mechanisms that apply only to WM for purely visual representations. Therefore, it is not essential to our conclusions that people relied exclusively on visual representations. Second, several studies have shown that controlling for verbal recoding of visual material in tasks similar to ours does not affect performance (e.g., Luck \& Vogel, 1997; Morey \& Cowan, 2004). Therefore, there was little to be gained from placing the extra burden of articulatory suppression on participants.

\section{Method}

Participants. Twenty-four students from a Swiss university participated in Experiment 1A. Their mean age was 24 years (range $=18$ to 30 ), and four of them were male. Participants received financial incentives (30 Swiss francs for approximately two hours) or partial course credit in exchange for their participation. For Experiment 1B, 60 participants (30 per group, 80\% female) were tested. The mean age was 23 years (range of predictable group $=18$ to 31 ; range of unpredictable group $=18$ to 33).

Task and stimuli. All participants were tested individually in a laboratory cabin. All experiments were programmed with the Psychophysics Toolbox (Brainard, 1997; Pelli, 1997) implemented in MATLAB. Memory stimuli (colored circles with a radius of $1.45 \mathrm{~cm}$ in Experiment 1A and of $0.95 \mathrm{~cm}$ in Experiment 1B) were presented at an eccentricity from the center of $8.8 \mathrm{~cm}$ in Experiment $1 \mathrm{~A}$ and of $5.9 \mathrm{~cm}$ in Experiment $1 \mathrm{~B}$.

Table 1

Predictions for Nonfocused Information in WM (Experiments $1 A$ and $1 B$ )

\begin{tabular}{|c|c|c|c|}
\hline \multirow[b]{2}{*}{ Factor } & \multicolumn{3}{|c|}{ Predictions for nonfocused information } \\
\hline & Maintenance & Weakening & $\begin{array}{c}\text { Removal } \\
\text { (flexible control) }\end{array}$ \\
\hline Effect of number of cues & 2 -cue $=1$-cue & 2 -cue $<1$-cue & 2-cue $=1$-cue \\
\hline Effect of predictability & Pred $=$ unpred & Pred $=$ unpred & Pred $>$ unpred \\
\hline \multicolumn{4}{|c|}{$\begin{array}{l}\text { Note. Overview of the predictions from three hypotheses (columns) for the effect of number of cues and } \\
\text { predictability of the number of cues in Experiments } 1 \mathrm{~A} \text { and } 1 \mathrm{~B} \text {. If nonfocused items are always maintained in } \\
\text { WM, no difference between single-cue and two-cue trials and between predictable and unpredictable conditions } \\
\text { is predicted. If nonfocused items are weakened by focusing one item, two-cue trials should be worse than } \\
\text { single-cue trials. Again, no effect of predictability is expected. If maintenance of nonfocused items is under } \\
\text { flexible control, there should be no difference between single-cue and two-cue trials (in the unpredictable } \\
\text { condition), and performance on single-cue trials should be better in the predictable than the unpredictable } \\
\text { condition. WM = working memory; Pred = predictable condition; Unpred = unpredictable condition. }\end{array}$} \\
\hline
\end{tabular}


Experiment 1A. Each trial began with a memory display consisting of six colored circles (randomly chosen from a set of nine colors), arranged on an imaginary circle centered on a black screen. The display remained on the screen for $1 \mathrm{~s}$ and was followed by an interval (see Figure 1 for interval lengths) during which the screen went black. After this interval, none, one, or two retro-cues were presented sequentially with an intercue interval of $500 \mathrm{~ms}$.

The cues were white central arrows, displayed for $100 \mathrm{~ms}$, pointing to one of the six circle locations from the memory display. The last presented cues were always valid in predicting the location to be probed later. In case of two-cue trials, the first and the second cue never pointed to the same location. Finally, a probe color was presented in the original location of one of the six colored circles. The task was to compare the color of the circle in the probe display to the color of the circle at the same location from the memory display. The probe matched the memory display circle on half the trials (match trials). On the other half of trials the probe was either a color from another location in the memory display or a new color (mismatch trials). The probe remained on screen until the participant answered by pressing the left arrow key for a match and the right arrow key for a mismatch. Performance feedback was immediately provided by a message on the screen (the German word for "right" or "wrong"). The feedback message disappeared after $500 \mathrm{~ms}$ and was followed by a gray intertrial interval of $1.5 \mathrm{~s}$. Speed of response was not emphasized. The predictability conditions were manipulated within subject across sessions by providing only single-cue and no-cue trials in the predictable condition and all cue types in the unpredictable condition (rows 1 to 5 in Figure 1).

To create conditions with matched retention intervals and to prevent participants from anticipating the number of cues in a trial, we manipulated the intervals between memory display and cue (memory-cue interval) as well as the intervals from the last cue to the probe (post-cue time). Our primary goal was to equate the overall retention interval, the time between the memory display and the valid cue, and the post-cue time for single-cue and two-cue trials; this was accomplished by the two conditions depicted in row 2 and row 3 of Figure 1. To prevent anticipation of the number of cues from the first memory display-cue interval, we also needed the conditions depicted in row 1 and row 4 in Figure 1.

In Experiment 1A, we used a long and a short no-cue condition. The long interval no-cue condition was matched to the overall retention time of the three cue conditions and served as a baseline for evaluating the retro-cue benefit for the cue conditions at a constant retention interval. In the short interval no-cue condition, the onset of the probe was matched to the onset of the cue in the early-onset single-cue condition. The comparison of the early single-cue and the short no-cue condition tests whether there is still a retro-cue benefit if we assume that retrieval in the cue condition

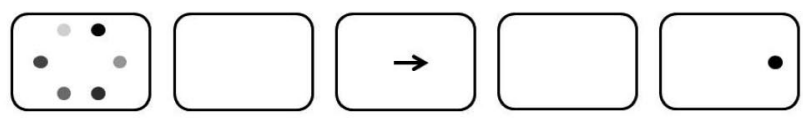

1 Cue (early)

\begin{tabular}{|l|l|l|l|l|}
\hline Memory Display & 1000 & cue & 1100 & Probe \\
\hline
\end{tabular}

1 Cue (late)

\begin{tabular}{|l|ll|l|l|}
\hline Memory Display & 1600 & cue & 500 & Probe \\
\hline
\end{tabular}

2 Cues

\begin{tabular}{|l|l|l|l|l|l|l|}
\hline Memory Display & 1000 & cue & 500 & cue & 500 & Probe \\
\hline
\end{tabular}

No Cue (long)

\begin{tabular}{|l|l|l|}
\hline Memory Display & 2200 & Probe \\
\hline
\end{tabular}

No Cue (short)

\begin{tabular}{|l|l|l|}
\hline Memory Display & 1000 & Probe \\
\hline
\end{tabular}

2 Cues (late onset)

\begin{tabular}{|l|l|l|l|l|l|l|}
\hline Memory Display & 1600 & cue & 500 & cue & 500 & Probe \\
\hline
\end{tabular}

Figure 1. Overview of the four experimental conditions in Experiment 1A (upper five rows) and in Experiment 1B (upper three rows and last row). The memory display was presented for $1 \mathrm{~s}$, and the probe display remained on screen until the participant entered the answer via the keyboard. Cues were presented for $100 \mathrm{~ms}$. The interval lengths in milliseconds are depicted in the corresponding intervals. Two predictability conditions were distinguished. In Experiment 1A, the predictable condition comprised both single-cue and both no-cue trial conditions (rows 1, 2, 4, and 5) and the unpredictable condition comprised all conditions of rows 1 to 5. In Experiment 1B, the predictable group comprised single-cue trials (upper two rows) and the unpredictable group comprised single- and two-cue trials (rows 1, 2, 3, and 6). In each trial, the last cue validly indicated which location was probed at the end of the trial. 
commences at the onset of the cue, whereas it starts at the onset of the probe in no-cue trials. In that case, the early-onset single-cue condition and the short no-cue condition are matched for their retention intervals.

Experiment 1B. The procedure of Experiment 1B differed from that in Experiment 1A regarding only two aspects: First, predictability was manipulated between subjects by creating two experimental groups. ${ }^{5}$ This was done to rule out carryover effects between the two predictability conditions. Second, we did not include no-cue trials. Instead, there was a two-cue late-onset condition, comparable to the two-cue condition in Experiment 1A, but the interval between memory display and first cue was 1,600 ms (see row 6 in Figure 1). With the inclusion of this condition, we prevented anticipation of the number of cues even for trials with a late cue onset. The durations of all intervals for each condition can be seen in Figure 1. Single- and two-cue conditions matching with regarding to overall retention interval, valid cue onset time, and post-cue time are depicted in rows 2 and 3 of Figure 1. The conditions depicted in rows 1 and 6 of Figure 1 served to prevent the anticipation of the number of cues from the first memory display-cue interval.

Procedure. For Experiment 1A, each participant completed 12 practice trials prior to each test session. Each of the two test session consisted of 480 trials (eight blocks). Predictability conditions were manipulated across the two sessions with counterbalanced order. In the unpredictable condition, one third of trials were no-cue trials (equal number for long and short retention interval), one third were single-cue trials (equal number for early and late cue onset) and the remaining third were two-cue trials. In the predictable condition, half of the trials were no-cue trials (equal number for long and short retention interval) and the other half were single-cue trials (equal number for early and late cue onset). For Experiment 1B, each participant completed one session with 24 practice trials and 320 test trials (eight blocks). There were equal numbers of trials from each condition in each experimental group; for the predictable group these were only the two kinds of single-cue trials, whereas for the unpredictable group these were single-cue trials and two-cue trials. Match and mismatch trials as well as the cue conditions were randomly intermixed in both experiments.

\section{Results}

For Experiment 1A, the average percentage correct across conditions was $77.2 \%(S D=8.9)$. The average percentage correct in Experiment $1 \mathrm{~B}$ was $81.4 \%(S D=6.3)$ in the predictable group and $81.5 \%(S D=6.8)$ in the unpredictable group. All analyses for both experiments were conducted on the dependent measure percentage correct.

Retro-cue benefit. To test whether valid retro-cuing improves performance, we compared the no-cue condition (long) to the cue conditions; these conditions were matched regarding the overall retention interval in Experiment 1A. All comparisons revealed significant retro-cue benefits, showing that retro-cuing improved performance compared to not providing a cue. Performance in the single-cue (late) condition was significantly better than in the no-cue condition, both in the predictable session, $t(23)=4.068$, $p<.001$, and in the unpredictable session, $t(23)=6.304, p<$ .001 . Performance for single-cue trials (early) was also better than for the no-cue condition, both in the predictable and in the unpredictable session, $t(23)=9.031, p<.001$, and $t(23)=6.173, p<$ .001 , respectively. Furthermore, performance in two-cue trials was significantly better than in no-cue trials (long), $t(23)=3.848, p=$ .001. Averages for each condition are shown in Figure 2, panel A.

Additionally, we compared the early single-cue condition to the no-cue condition (short) in Experiment 1A. This comparison provides a more conservative test of the retro-cue benefit, because it equates the time between memory display and probe in the no-cue condition with the time between memory display and cue in the retro-cue condition (see rows 1 and 5 in Figure 1). Thus, these two conditions are equated for the uncued retention interval; that is, the time during which the entire memory set had to be retained in WM, without any information about which memory contents will be relevant at test. This comparison revealed a significant retro-cue benefit, for the predictable condition, $t(23)=7.368, p<.001$, and for the unpredictable condition, $t(23)=4.194, p<.001$.

Predictability. To examine whether predictability influences WM performance, we conducted a within-subject analysis of variance (ANOVA) with the factors predictability (predictable and unpredictable condition) and cue onset (early or late) for singlecue trials for Experiment 1A (see Figure 2, panel A). Early cue onset led to better performance than late cue onset, $F(1,23)=$ 26.967, $p<.001$, partial $\eta^{2}=.540$. The predictable and the unpredictable conditions did not differ significantly from each other, $F(1,23)=1.633, p=.214$, partial $\eta^{2}=.066$, and the interaction between the two variables was nonsignificant, $F(1,23)=2.144, p=.157$, partial $\eta^{2}=.085$. Experiment $1 \mathrm{~B}$ confirmed this pattern of results for the between-subjects design: Performance was better for early single-cue onset than for late single-cue onset, $F(1,58)=35.818, p<.001$, partial $\eta^{2}=.382$. There was no significant effect of predictability, $F(1,58)=1.557$, $p=.217$, partial $\eta^{2}=.026$, and no significant interaction, $F(1,58)=2.717, p=.105$, partial $\eta^{2}=.045$. Figure $2($ panel $\mathrm{B})$ shows the averages for each experimental group (predictability) and cue onset condition.

Nonfocused information in WM. To test whether noncued information remains accessible in WM after cuing other information, we conducted a paired $t$ test, comparing performance in late single-cue trials to performance in (early) two-cue trials for the unpredictable condition (rows 2 and 3 in Figure 1). These two conditions were matched with regard to the onset time of the valid cue, the post-cue time, and overall retention time. Therefore the only difference between these two kinds of trials is that the late cue is preceded by an earlier cue in the two-cue trials. If focusing on that earlier cued item led to impairment of memory for the remaining items, as predicted by the slot-and-averaging model and the resource model, the two-cue trials should result in worse performance than should the late single-cue trials.

There was no statistically significant difference between the two kinds of trials. In Experiment 1A, mean accuracy in single-cue trials (late onset) was $78.6 \%(S D=10.3)$, and mean accuracy in two-cue trials was $77.2 \%(S D=9.3), t(23)=1.018, p=.319$ (see Figure 3, panel A for means). Experiment $1 \mathrm{~B}$ confirmed this

\footnotetext{
${ }^{5}$ Due to a programming error, red color probes appeared only in match and never in mismatch trials. Analyses with and without red probe trials converge. Analyses excluding red probe trials are reported.
} 

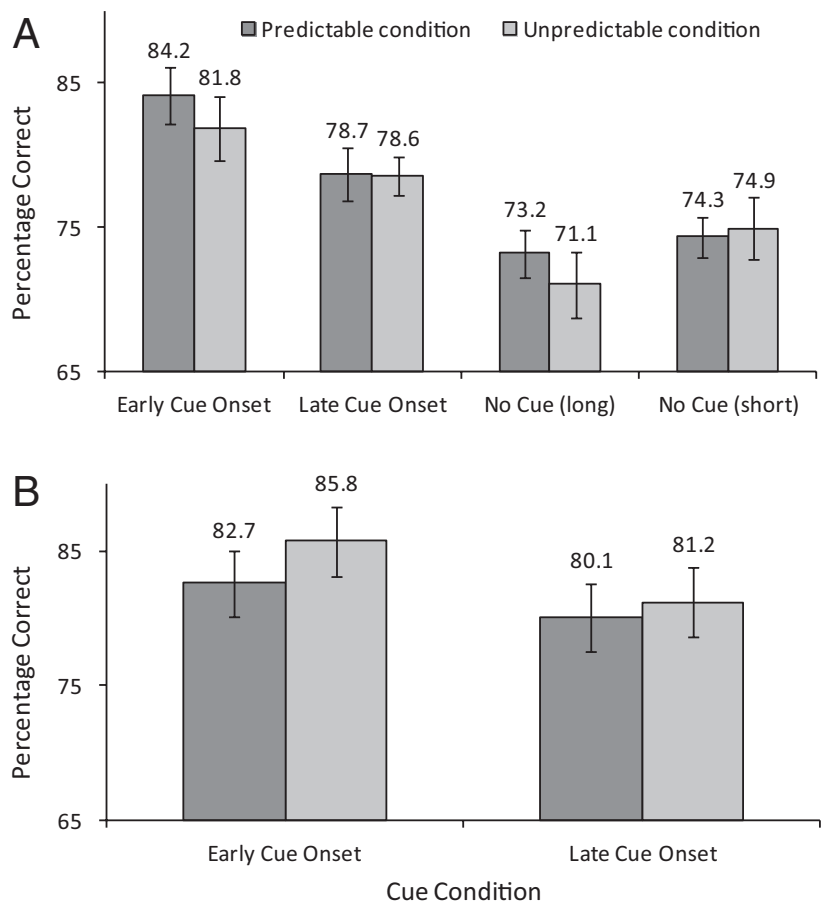

Figure 2. Panel A shows the percentage correct for single-cue and no-cue trials for Experiment 1A for the predictable and unpredictable conditions. Error bars represent within-subject confidence intervals (95\%). Panel B shows the percentage correct for single-cue conditions for Experiment 1B for the predictable and unpredictable groups. Error bars represent betweensubjects confidence intervals $(95 \%)$.

pattern: Late single-cue trials and early two-cue trials in the unpredictable group did not differ significantly from each other (see Figure 3, panel B for means), $t(29)=0.733, p=.469$. These findings imply that while attention is focused on the first-cued item, the noncued information remains unimpaired and can be focused on when a second cue follows. An additional comparison of the two-cue condition to the early single-cue condition (Figure 1 , rows 1 vs. 3 ), not matched with regard to time parameters, yielded significant differences in both experiments: Performance in the early single-cue condition was significantly better than in the two-cue condition, $t(23)=3.306, p=.003$ for Experiment $1 \mathrm{~A}$, and $t(29)=6.334, p<.001$ for Experiment 1B.

\section{Discussion}

Experiment 1A showed that valid cue trials yield better performance than trials without any cuing, thus replicating the retro-cue benefit for our experimental procedure. This finding shows that our retro-cues were used to direct attention to WM representations.

Our finding of equivalent performance for single-cue and twocue trials for matched retention intervals shows that focusing attention in WM on a single representation does not lead to forgetting of the other representations held in WM, supporting the maintenance hypothesis (see Table 1). The finding of better performance for early single-cue trials than for late single-cue trials and for early two-cue trials suggests forgetting as a function of time. Worse memory after longer retention intervals has been observed in some previous studies of WM for colors (Morey \& Bieler, 2012; Zhang \& Luck, 2009; but see Magnussen \& Greenlee, 1999) and could be attributed to temporal distinctiveness (Shipstead \& Engle, 2012). In the present context it is important that this time-related decline of accuracy occurs independently of cuing and focusing. When singlecue and two-cue trials were matched for the duration of the retention interval, accuracy did not differ.

The result that time-matched trials with single and two cues lead to the same performance is in line with the prediction from the threeembedded-components model (Oberauer, 2002, 2009) and furthermore in agreement with the results of Landman et al. (2003), who did not find any significant differences between single-cue and two-cue trials in a similar experiment with a shorter time frame. This outcome indicates that the focus of attention can be flexibly reoriented in WM and that focusing on one item does not compromise retention of the remaining items. However, the results challenge fixed-capacity and constant-resource theories, which predict that focusing one item reduces the number of slots or the amount of resource available for noncued, nonfocused WM contents.

Our result is in apparent contrast to the one reported by Woodman and Vecera (2011), who found that performance declined over successive probes testing different items of a memory set. The reasons for this discrepancy could be that in our experiments people merely focused on several items in succession, whereas in the study of Woodman and Vecera, they were probed for overt responses on
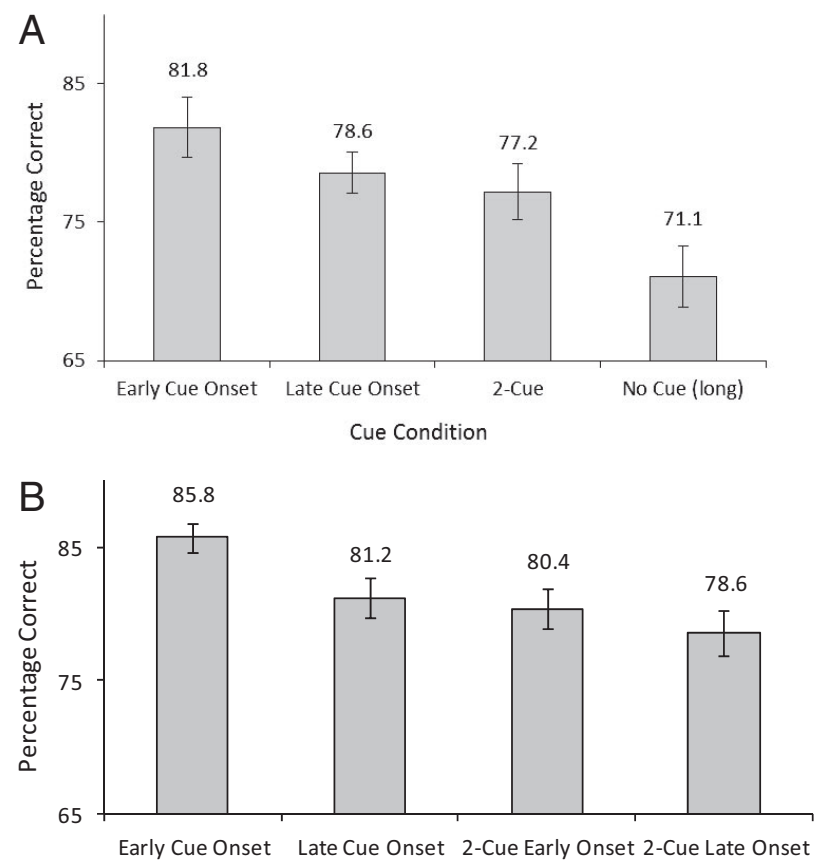

Cue Condition

Figure 3. Panel A shows the percentage correct for single-cue trials (early and late onset), the two-cue condition, and the matched no-cue condition in Experiment 1A (unpredictable condition). Error bars represent within-subjects confidence intervals (95\%). Panel B depicts accuracy for single-cue trials (early and late onset) and two-cue trials (early and late onset) in Experiment 1B (unpredictable condition). Error bars represent between-subjects confidence intervals $(95 \%)$. 
several items in succession. Probing memory for overt responses is known to generate output interference (Cowan, Saults, Elliott, \& Moreno, 2002; Oberauer, 2003). Our finding that memory survives two successive retro-cues with no measurable impairment suggests that merely focusing on an item does not create interference in the way that retrieving an item for an overt response does.

We found no effect of predictability of the number of cues, neither in the within-subject nor in the between-subjects design. In single-cue trials, participants in the predictable session or group could have reduced WM load by removing the noncued items from central WM; in the unpredictable session or group, this strategy would have been detrimental. Nevertheless, performance in the two conditions was indistinguishable for single-cue trials. Together with the finding of equivalent performance in single- and two-cue trials, this implies that noncued information was not removed but maintained in the predictable condition (for an overview of predictions, see Table 1), even when it was known to be irrelevant (cf. Makovski \& Jiang, 2008). This finding goes against the assumption in the three-embeddedcomponents model (Oberauer, 2001, 2002) that information can be removed from the central components of WM when it is known to be irrelevant for the task.

It is conceivable that voluntary removal (as an indicator of flexible control) is a slow process that needs more time than was available to our participants. However, experiments on removal of information from verbal WM (Oberauer, 2001, 2002) indicate that it takes little more than $1 \mathrm{~s}$ to remove an irrelevant set from the central component of WM. In the early single-cue trials, participants had more than $1 \mathrm{~s}$ between the cue and the probe to remove irrelevant WM contents. This should have been sufficient time for removal, and yet we obtained no evidence for it. Hence, our finding suggests that noncued information remains in WM, at least for the duration of our post-cue time.

Within the framework of null-hypothesis testing, any support for the null hypothesis is necessarily indirect, stemming from a failure to support the alternative hypothesis. A direct assessment of the strength of evidence for the null hypothesis can be derived from likelihood ratios by estimating how much more likely it is to obtain the observed data under the assumptions of the null hypothesis compared to an alternative hypothesis (Glover \& Dixon, 2004). In addition, the strength of evidence for the null hypothesis can also be assessed by the Bayes factor. From the Bayes factor we can calculate the posterior probability that the null hypothesis is true, given the data, under the assumption of equal prior probabilities for the null hypothesis and the alternative hypothesis (Masson,
2011). In Table 2, we provide likelihood ratios, Bayes factors, and posterior probabilities of the null hypothesis for the comparison of predictability conditions and the comparison of single-cue and two-cue trial performance, separately for Experiment $1 \mathrm{~A}$ and $1 \mathrm{~B}$. In all cases, the data consistently support the null hypothesis over the alternative hypothesis. In the case of the predictability comparison, the support for the null hypothesis is actually stronger than the likelihood ratios and Bayes factors suggest, because in the two experiments the deviation from the null hypothesis went in different directions. Thus, we are testing the null hypothesis against two different, mutually contradictory alternative hypotheses in Experiments $1 \mathrm{~A}$ and $1 \mathrm{~B}$.

Our results suggest that WM representations can be maintained without continuous focal attention. This finding is in accordance with the three-embedded-components framework of WM (Oberauer, 2002, 2009). It is also in accordance with several computational models of WM, such as the feature model by Nairne (1990), the primacy model by Page and Norris (1998) and the serial order in a box (SOB) model by Farrell and Lewandowsky (2002; Oberauer \& Lewandowsky, 2008; Oberauer et al., 2012), all of which assume that maintenance in WM does not require focal attention.

\section{Experiment 2: Defocused Information in WM-The Intrusion Approach}

The first experiments showed that information that has never been focused after initial encoding is not impaired by focusing other information in WM. However, the case might be different for defocused items in WM. These items gained a special status once by being focused during the retention interval but were then deprioritized again while another item is prioritized. We consider three hypotheses about the fate of defocused items: the back-tobaseline hypothesis, the refreshing hypothesis, and the inhibition hypothesis. All of them are motivated by prior theorizing or data.

The back-to-baseline hypothesis is that defocused items are set back to their status before being focused. Thus, their memory strength would not differ from that of other, never cued items. Bays and Husain (2008) investigated accuracy in a visual WM task as a function of the history of saccades to the locations of memory items during the retention interval. They observed a benefit for the last fixated item compared to previously fixated objects but no advantage for any object fixated before the last. If fixating is an indicator of attentional focusing, this result would suggest that

Table 2

Likelihood Ratios in Favor of the Null Hypotheses for Experiments $1 \mathrm{~A}$ and $1 \mathrm{~B}$

\begin{tabular}{lccccc}
\hline \multicolumn{1}{c}{ Comparison } & $n$ & $k 1(k 2)$ & $\mathrm{LR}_{\mathrm{BIC}}$ & $\mathrm{BF}$ & $\mathrm{P}_{\mathrm{BIC}}(\mathrm{HOID})$ \\
\hline E1A & & & & & \\
$\quad$ Predictable vs. unpredictable condition & 24 & $2(3)$ & 2.1505 & 2.1591 & .6835 \\
$\quad$ Single- vs. two-cue trials & 24 & $2(3)$ & 2.8874 & 2.8910 & .7430 \\
E1B & & & & & \\
$\quad$ Predictable vs. unpredictable group & 60 & $2(3)$ & 3.5470 & 3.5143 & .7785 \\
$\quad$ Single- vs. two-cue trials & 30 & $2(3)$ & 4.1584 & 4.1709 & .8066 \\
\hline
\end{tabular}

Note. $n=$ number of participants; $k 1=$ number of free parameters for null hypothesis $(\mathrm{H} 0) ; k 2=$ number of free parameters for alternative hypothesis $(\mathrm{H} 1) ; \mathrm{LR}_{\mathrm{BIC}}=$ likelihood ratio in favor of the null hypothesis, corrected for number of free parameters according to Bayesian information criterion; $\mathrm{BF}=$ Bayes factor (according to Masson, 2011); $\mathrm{P}_{\mathrm{BIC}}(\mathrm{HOID})=$ posterior probability for the null hypothesis (Masson, 2011). 
previously focused objects return to baseline level, in line with the back-to-baseline hypothesis.

The refreshing hypothesis states that focusing on an item in WM could leave that item strengthened after the focus moved away. This possibility is implied by the notion of attention-based refreshing (Johnson et al., 2005; Raye, Johnson, Mitchell, Greene, \& Johnson, 2007). Refreshing is assumed to be a mechanism strengthening a representation in memory by briefly thinking of it. Importantly, this happens in WM without perceptual input (Johnson, 1992; Johnson \& Hirst, 1993). Hence, according to the refreshing hypothesis, by focusing a representation in WM (i.e., refreshing it), its activation and presumably its binding to its context (e.g., its spatial location) are strengthened. In the context of a recognition test as in our experiments, it makes a difference whether refreshing only increases an item's activation or additionally strengthens bindings to its context, as we explain below. In the data of Bays and Husain (2008), though the effect was not significant, there was a trend toward better accuracy for the next-to-last fixated object compared to other objects. Possibly a lack of power prevented this trend, which would be predicted from the refreshing hypothesis, from becoming significant.

The inhibition hypothesis states that defocused information is suppressed or inhibited relative to other items in WM to facilitate disengagement of the focus of attention. Several researchers have assumed that inhibition is important to overcome prepotent responses and to prevent getting stuck in a task (Koch, Gade, Philipp, \& Schuch, 2010; Mayr \& Keele, 2000) or on an item (Klein, 2000; Maxcey-Richard \& Hollingworth, 2012; Pratt, Kingstone, \& Khoe, 1997). Phenomena such as inhibition of return (Klein, 2000) and response suppression (Henson, 1998; Hübner \& Druey, 2006) suggest that leaving a once-selected representation behind is often accompanied by the suppression of that representation. Bao, Li, Chen, and Zhang (2006) have proposed that inhibition of defocused items serves to facilitate focus switching in WM. In case defocused information is inhibited, its representation is less accessible after defocusing than are representations of nonfocused information. ${ }^{6}$

We used the same basic paradigm as in Experiments $1 \mathrm{~A}$ and $1 \mathrm{~B}$ with some variations. The probe stimulus was presented centrally and had to be compared to the last cued object. The probe either matched or mismatched the cued item. We distinguish different classes of mismatching probes: New probes are objects that have not been presented in the memory display. Intrusion probes are objects that were included in the memory display but in a different location than the one last cued. Accordingly, they require a rejection. Intrusion probes can be created by presenting either a noncued object or the previously cued (i.e., defocused) object. We investigate whether rejecting previously cued probes is harder or easier than rejecting new probes or noncued intrusion probes and infer from this whether defocused information is maintained, strengthened, or inhibited in WM.

To make predictions for the three possible fates of defocused information, we need to consider the processes involved in recognition; namely, familiarity and recollection processes. The strength of the familiarity signal is sufficient to reject a new item probe as a mismatch due to its weak familiarity. In contrast, familiarity is not sufficient to reject intrusion probes. Intrusion probes are as familiar as matching probes. Recollection of item-location bindings is needed to discriminate between matching probes and in- trusion probes. Because recollection is slower than the accrual of familiarity over time (Göthe \& Oberauer, 2008), we expect that rejection of intrusion probes is slower than rejection of new probes.

On the basis of these considerations, we make the following predictions for the three hypothetical consequences of defocusing: If the defocused item was simply maintained in a state equal to that before being focused, responses to previously cued intrusion probes should not differ from responses to noncued intrusion probes. If the defocused item was suppressed in WM, rejecting a probe matching that item should be easier than rejecting an intrusion probe matching one of the noncued items, because the suppressed items would elicit a weaker familiarity signal. As a result, the correct-rejection rate of defocused items should be higher than the correct-rejection rate for noncued intrusion probes. Because the familiarity signal is available quickly, RTs should also be faster for correct rejections than for noncued intrusion probes.

In case defocused information is strengthened in WM, two possibilities can be distinguished: One is that defocused items remain in WM with increased activation but without strengthening the bindings to their spatial locations. Their high activation would lead to strong familiarity, resulting in an increase of false alarms to these probe types and slow RTs for correct rejections. The alternative possibility is that the bindings of the defocused item to its location are strengthened, or that this item is bound to a representation of the fact that it had been defocused before. Recollection of this binding information would facilitate "recall to reject" (Rotello \& Heit, 1999): The defocused item can be recollected together with its context and thereby be identified as not being the item in the currently cued location. This would enable participants to correctly reject defocused intrusion probes with higher accuracy than noncued intrusion probes. However, because recall-to-reject relies on recollection, these responses are predicted to be slower than rejection of new probes.

In addition to manipulating the mismatching probe types, we varied the time interval after the second cue (post-cue time; see Figure 4), to investigate whether it takes time to fully use the cue.

\section{Method}

Participants. Thirty-seven students participated in this experiment. Their mean age was 22 years (range $=19$ to 28 ), and nine of them were male. Participants completed two sessions scheduled on different days and received financial compensation (30 Swiss francs for two 1-hr sessions) or partial course credit in exchange for their participation. One participant was excluded from analysis due to technical problems.

\footnotetext{
${ }^{6}$ Inhibition of the defocused item must be distinguished from removal of all irrelevant items. The inhibition hypothesis as formulated here assumes that the defocused item is weakened relative to other, never focused items. Removal of irrelevant items would imply that, once the second (and last) cue is presented, all items except the last-cued one are removed because they are known to be irrelevant. This includes the defocused item, but removal would not render the defocused item weaker than other, nonfocused items. Our three hypotheses about the fate of defocused information concern the comparison of defocused to nonfocused items within the set of items that are irrelevant after the second cue. Therefore, these three hypotheses are orthogonal to the assumption of removal.
} 

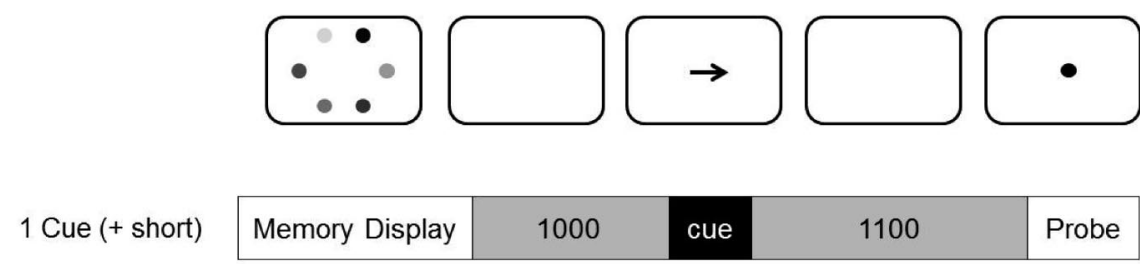

\begin{tabular}{|c|c|c|c|c|c|}
\hline 1 Cue (+ long) & Memory Display & 1000 & cue & 1600 & Probe \\
\hline
\end{tabular}

\begin{tabular}{|c|c|c|c|c|c|c|c|}
\hline 2 Cues (+ short) & Memory Display & 1000 & cue & 500 & cue & 500 & Probe \\
\hline
\end{tabular}

\begin{tabular}{|c|c|c|c|c|c|c|c|}
\hline 2 Cues (+ long) & Memory Display & 1000 & cue & 500 & cue & 1100 & Probe \\
\hline
\end{tabular}

Figure 4. Overview of the experimental design and conditions for Experiment 2. The first two rows show the two single-cue trial conditions (with short and long post-cue time), and the third and fourth rows show the two-cue trial conditions (short and long post-cue time).

Task and stimuli. The same color-recognition task as in Experiment 1B was used with some variation. Only single- and two-cue trials were provided. Unlike in Experiment 1, where a location-bound probe was presented, the probe was presented centrally and had to be compared to the color of the last cued object. Thereby, the importance of processing the cue was stressed. A correct response was possible only if the cue was used to identify the relevant item. Of special interest were mismatch trials. These were created by different probe types: The probe was a new color (not presented in the memory display), a noncued color (either a spatial neighbor or a nonneighbor to the last cued location), or the color of the previously cued circle. Fifty percent of trials were positive probes (matches). The remaining mismatch trials were split into $25 \%$ new color probes and $25 \%$ intrusion probes. For single-cue trials, half of the intrusion trials were spatial neighbors and half non-neighbors to the last cued item. For two-cue trials, the intrusion trials were split into $12.5 \%$ previously cued item probes and $12.5 \%$ noncued probes. Of the noncued probes, half were spatial neighbors of the last cued item. ${ }^{7}$

Additionally, the time interval between memory display and first cue was kept constant, and only the interval after the last cue (post-cue time) was varied: There was a short interval of $1,100 \mathrm{~ms}$ for single-cue trials and $500 \mathrm{~ms}$ for two-cue trials, and there was a long interval of 1,600 ms for single-cue trials and 1,100 ms for two-cue trials (see Figure 4). With this manipulation we tested whether it takes time to make full use of the retro-cues. The short and long post-cue intervals differed between single-cue and twocue trials because we held overall retention interval constant between these two kinds of trials.

Procedure. Each participant completed 34 practice trials prior to the first test session and four practice trials prior to the second session. Each test sessions included 416 trials, split into nine blocks (the first eight including 50 trials each and the last one including 16 trials). There number of trials was equal in the four cue conditions. Cue conditions and probe types were randomly intermixed. Participants were instructed to respond as accurately and as fast as possible.

\section{Results}

Participants completed on average $82.5 \%$ of trials correctly $(S D=6.4)$. Percentage correct and RT data from the different categories of mismatch trials served as dependent variables. The data from the match trials are included in the analysis of post-cue time but are omitted for analysis of the probe types. The mean scores and the corresponding standard deviations for each condition can be found in the Appendix.

Mismatch trials were sorted into three (single-cue trials) or four (two-cue trials) probe type categories: new, non-neighbor intrusion, neighbor intrusion, and previously cued intrusion probes (the latter only for two-cue trials). Trials associated with responses faster than $100 \mathrm{~ms}$ and longer than $7 \mathrm{~s}$ were excluded from the RT analyses, as were error trials. RTs were log-transformed for all analyses to reduce the skew of the distributions. Untransformed RT means are plotted in the figures and reported in the text to facilitate readability. ${ }^{8}$ For some analyses of variance, the sphericity assumption was violated. In these cases, corrected GreenhouseGeisser degrees of freedom (recognizable by noninteger values) are reported. For a graphical display of results, see Figure 5.

Single-cue trials. An ANOVA with probe type (new, nonneighbor intrusion, and neighbor intrusion probe) as the independent variable was run on accuracy data (percentage correct) for

\footnotetext{
${ }^{7}$ Due to a programming error, participants had on average 2.4 previously cued probe trials more and 2.4 neighbor item probe trials less in Conditions 3 and 4 than originally intended.

${ }^{8}$ The same trimming procedure was used as for the log-transformed RTs. Additionally, RTs exceeding the participant's mean per design cell by more than three standard deviations were excluded.
} 


\section{SINGLE-CUE TRIALS}


TWO-CUE TRIALS
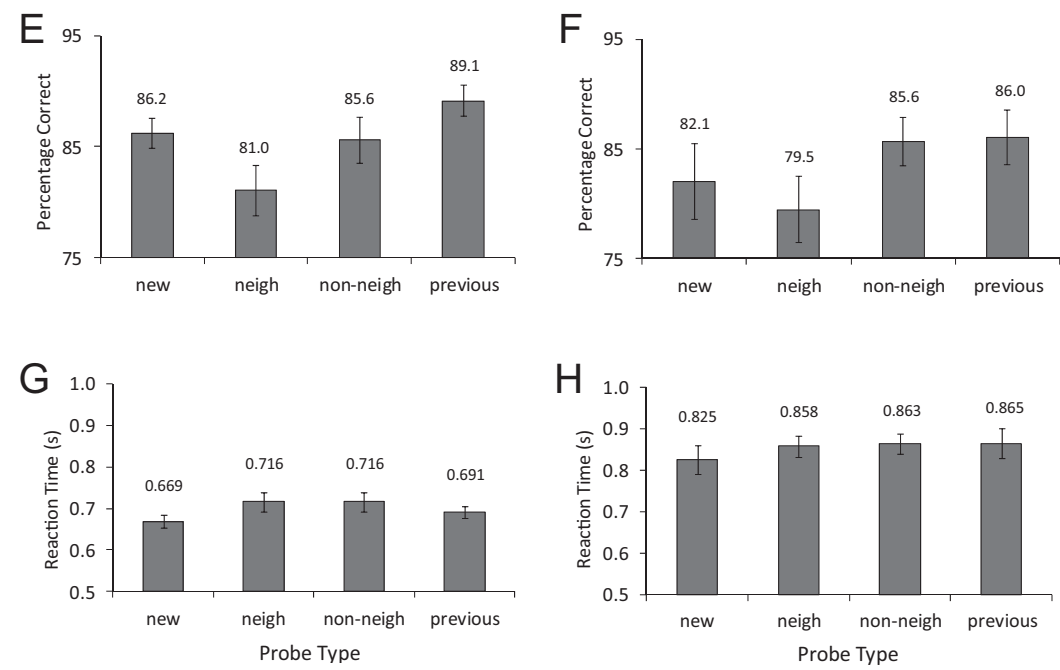

Figure 5. Results for Experiment 2 (first column) and Experiment 3 (second column). The first row shows accuracy data for Experiment 2 (panel A) and Experiment 3 (panel B), and the second row shows RT data for Experiment 2 (panel C) and Experiment 3 (panel D) for single-cue trials. The third row shows accuracy data for Experiment 2 (panel E) and Experiment 3 (panel F), and the fourth row shows reaction time data for Experiment 2 (panel G) and Experiment 3 (panel $\mathrm{H}$ ) for two-cue trials. In each graph, the dependent variables are presented for each of the mismatch probe conditions (levels on the $x$-axis). Error bars represent within-subject confidence intervals (95\%).

single-cue trials. The main effect was significant, $F(2,70)=$ $11.98, p<.001$, partial $\eta^{2}=.255$. Planned contrasts revealed that new-item probes did not differ significantly from intrusion probes (i.e., neighbor and non-neighbor probes combined), $F(1,35)=$ 2.701, $p=.109$, partial $\eta^{2}=.072$. Neighbor probes led to significantly worse performance than non-neighbor probes, $F(1$, $35)=21.005, p<.001$, partial $\eta^{2}=.375$.

The same ANOVA was run on log-transformed RT data. The main effect again was significant, $F(2,70)=3.375, p=.040$, partial $\eta^{2}=.088$, reflecting faster RTs for new probes than for the two kinds of intrusion probes combined, $F(1,35)=5.674, p=$ .023 , partial $\eta^{2}=.139$. Neighbor and non-neighbor probes did not differ significantly from each other, $F(1,35)=2.026, p=.163$, partial $\eta^{2}=.055$.

Two-cue trials. In two-cue trials, mismatches can be created by new color, non-neighbor color, neighbor color, and previously cued color probes. There was a main effect of probe type on accuracy, $F(2.085,72.969)=13.041, p<.001$, partial $\eta^{2}=.271$. We again parsed the probe-type effect by a set of planned con-

\footnotetext{
${ }^{9}$ Previously cued probes were themselves neighbors and non-neighbors of the validly cued probe with a chance of 2:3. The categories of neighbors and non-neighbors excluded previously cued probes.
} 
trasts. New item probes did not differ significantly from all intrusion probes combined, $F(1,35)=1.466, p=.234$, partial $\eta^{2}=$ .040. Previously cued intrusion probes were more accurately rejected than neighbor and non-neighbor intrusion probes combined, $F(1,35)=37.304, p<.001$, partial $\eta^{2}=.516 .{ }^{9}$ Accuracy for neighbor probes was poorest and was significantly worse than that for non-neighbor probes, $F(1,35)=6.774, p=.013$, partial $\eta^{2}=$ .162 .

The overall effect of probe type on log-transformed RT was not significant, $F(2.346,82.110)=1.055, p=.361$, partial $\eta^{2}=.029$. Nevertheless, planned contrasts revealed that new color probes led to significantly shorter RTs than the three kinds of intrusion probes combined, $F(1,35)=5.765, p=.022$, partial $\eta^{2}=.141$. Previously cued intrusion probes did not differ from neighbor and non-neighbor probes combined, which in turn did not differ significantly from each other, both $F \mathrm{~s}<.004, p \mathrm{~s}>.95$, partial $\eta^{2}<$ .001 . A paired $t$ test showed that previously cued probes were rejected more slowly than new probes, $t(35)=2.425, p=.021$.

Post-cue time. We examined the effect of post-cue time through paired $t$ tests on accuracy and on RT, separately for singleand two-cue trials. Table 3 shows the relevant means and test statistics. There was no effect of post-cue time on accuracy, but participants answered significantly faster after a longer post-cue time. This result provides tentative evidence that it takes time to make fully efficient use of the retro-cue.

\section{Discussion}

In Experiment 2 we investigated what happens to defocused representations. The results showed that previously cued item probes were rejected better than other intrusion probe types. They were responded to about as fast as other intrusion probes and slower than new probes.

This pattern of effects can be explained within the dual-process theory of short-term recognition, distinguishing familiarity and recollection (McElree \& Dosher, 1989; Oberauer, 2008). New probes elicit a weak familiarity signal, indicating that the object has not been encountered recently. This weak familiarity signal offers a shortcut: Because the probe appears unfamiliar, it was most likely not the validly cued representation. Hence, new probes can be rejected on the basis of a familiarity process, which is assumed to be fast (Göthe \& Oberauer, 2008; Yonelinas, 2002). Intrusion probes elicit a stronger familiarity signal (due to having been encoded into WM recently) and therefore require a more time-consuming recollection process that retrieves information about which color was where in the memory display to reach a correct rejection. In line with the predictions of the dual-process theory, intrusion probes-and among them the previously cued probes-exhibited slower RTs than did new item probes.

The observation that previously cued probes were processed slowly but that accuracy on them was better than on the other mismatch probe types suggests recall-to-reject: The previously cued item is maintained in WM after it has been defocused, and the binding to its position remains strengthened. When the probe matches the previously cued item, it elicits a strong familiarity signal, which prevents fast rejection on the basis of low familiarity alone. Recollection reveals that the probe was part of the memory display but not in the relevant (i.e., last-cued) position. This information is available with high accuracy because of the strengthened item-position binding due to having been focused before. Therefore, previously focused probes can be rejected with high accuracy.

Neighbor probes were harder to reject than non-neighboring intrusion probes. This finding suggests that representations in WM are spatially imprecise (cf. Makovski \& Jiang, 2008), such that when one tries to focus on an item in a given location, neighboring items cannot be completely excluded. The notion of a spatially imprecise WM assumes that representations of spatially separated objects in WM are not perfectly distinct. Rather, each object is bound to its location in space, and the location representations overlap as a function of their proximity. When one location is cued, the location is used as a retrieval cue to the object bound to it. Because of location overlap, neighboring objects might be partially retrieved into the focus of attention, thereby causing interference from spatially close objects. A probe matching a neighbor of the cued item therefore matches part of the information in the focus, biasing the decision for neighbor probes toward a match response, although a rejection is required. Evidence for spatially imprecise representations in visual WM was also obtained by Schmidt, Vogel, Woodman, and Luck (2002), using invalid location cues: They cued an item's location and tested memory for the item in the cued location, a neighboring location, or a non-neighboring location. Memory for neighboring locations was better than for non-neighboring locations, as would be expected if the effect of cuing spilled over to neighbors of the cued item. In contrast to Schmidt et al., we never tested neighboring items directly but presented them as intrusion probes. In this role, the heightened availability of neighbors of the cued item led to worse performance because it rendered neighboring intrusion probes hard to reject. The increased difficulty of rejecting neighbor probes contrasts with the improved rejection for defocused probes. This contrast rules out any explanation of the two effects by the same process: It cannot be the case that both the defocused item

Table 3

Mean RTs (in Seconds) and Accuracies (in Percent Correct) in Experiment 2 as a Function of Post-Cue Interval

\begin{tabular}{lccr}
\hline \multicolumn{1}{c}{ Condition } & Short post-cue time & Long post-cue time & \multicolumn{1}{c}{$t(35), p$} \\
\hline RT, single-cue trials & $0.59(0.15)$ & $0.58(0.14)$ & $3.36, p=.002$ \\
RT, two-cue trials & $0.70(0.20)$ & $0.61(0.17)$ & $13.94, p<.001$ \\
Accuracy, single-cue trials & $84.4(6.6)$ & $84.4(6.6)$ & $0.05, p=.96$ \\
Accuracy, two-cue trials & $80.3(7.0)$ & $80.9(7.0)$ & $0.90, p=.33$ \\
\hline
\end{tabular}

Note. Standard deviations are given in parentheses. $\mathrm{RT}=$ reaction time. 
and the neighboring items receive a larger share of the WM resource than other items, are more highly activated in long-term memory, or are held in the focus of attention together with the validly cued item. We return to this issue in the General Discussion.

In sum, the results provide initial support to the refreshing hypothesis, according to which focusing an item strengthens its binding to its spatial location, thereby enabling recall-to-reject. However, an alternative explanation for the high accuracy on probes matching defocused items could be based on the inhibition hypothesis, assuming that the previously cued item is inhibited to prevent interference. This in turn should make rejection of a previously cued item easier, because a probe matching an inhibited item generates less familiarity than a probe matching a noncued item. The fact that previously cued items yielded rather slow RTs does not sit comfortably with the inhibition hypothesis, because a probe with low familiarity should be rejected quickly, as was the case for new probes. To be made compatible with the inhibition hypothesis, the RT data pattern would have to be explained by a speed-accuracy trade-off acting specifically on previously cued probes. This explanation appears unlikely, because it requires a paradoxical assumption: The presumed speed-accuracy trade-off would have to occur selectively for previously cued probes. Thus, the cognitive system would first have to identify a probe as matching a previously cued item and then shift the speed-accuracy criterion for the process of identifying whether or not the probe matches the last-cued item. The absurdity of this notion renders an inhibition hypothesis of the present data implausible. Nevertheless, because of the remaining ambiguity of how to interpret the high rejection accuracy for previously cued probes, we carried out a further experiment to explicitly test whether defocused items are inhibited or strengthened.

\section{Experiment 3: Defocused Information in WM-The Backshift Approach}

Experiment 3 serves to decide between the refreshing and the inhibition hypotheses. We presented up to three cues in succession.
This enabled us to investigate sequences in which the focus of attention was first cued to one item, then away from it, and finally back to it (sequence $\mathrm{ABA}$ ), in comparison to three-cue trials in which three different items were cued (sequence CBA). In both trials, the last-cued item $\mathrm{A}$ is indicated as relevant for the comparison with the probe. In the ABA sequence, that item has been previously cued, whereas in the CBA sequence it has not. If focusing on an item strengthens its representation in WM, the comparison of the probe should be more successful in trials with cuing sequence $\mathrm{ABA}$ than in trials with the CBA sequence. In contrast, if a previously cued item is inhibited, comparison of the probe to the finally cued item (A) should be impaired in the ABA sequences relative to the CBA sequences (see Mayr \& Keele, 2000 , for a demonstration of inhibition of previously used task sets based on this rationale).

\section{Method}

Participants. Twenty-seven students participated in the experiment. Their mean age was 26 years (range $=21$ to 35 ), and nine of them were male. Participants received financial compensation or course credit. One participant was excluded due to accuracy below the predefined exclusion criterion (65\% correct).

Task and stimuli. The same basic paradigm was used as in Experiment 2 with some adaptations (see Figure 6 for an overview of the flow of events in each condition, including interval durations). In the interval between memory display and probe display, one, two, or three cues were presented sequentially with an intercue interval of $700 \mathrm{~ms}$. The task was to compare the central probe to the item that was cued last in the trial. The probe could be either a match or a mismatch. Mismatches were classified into new-color probes, neighbor, non-neighbor, and previously cued probes. In three-cue trials, we did not distinguish neighbors and nonneighbors but rather collapsed them into one category: noncued item probes. Three-cue trials consisted of CBA and ABA sequences. The CBA sequences cued three different locations selected at random without replacement, whereas in the ABA sequence the first and the third cue pointed to the same location

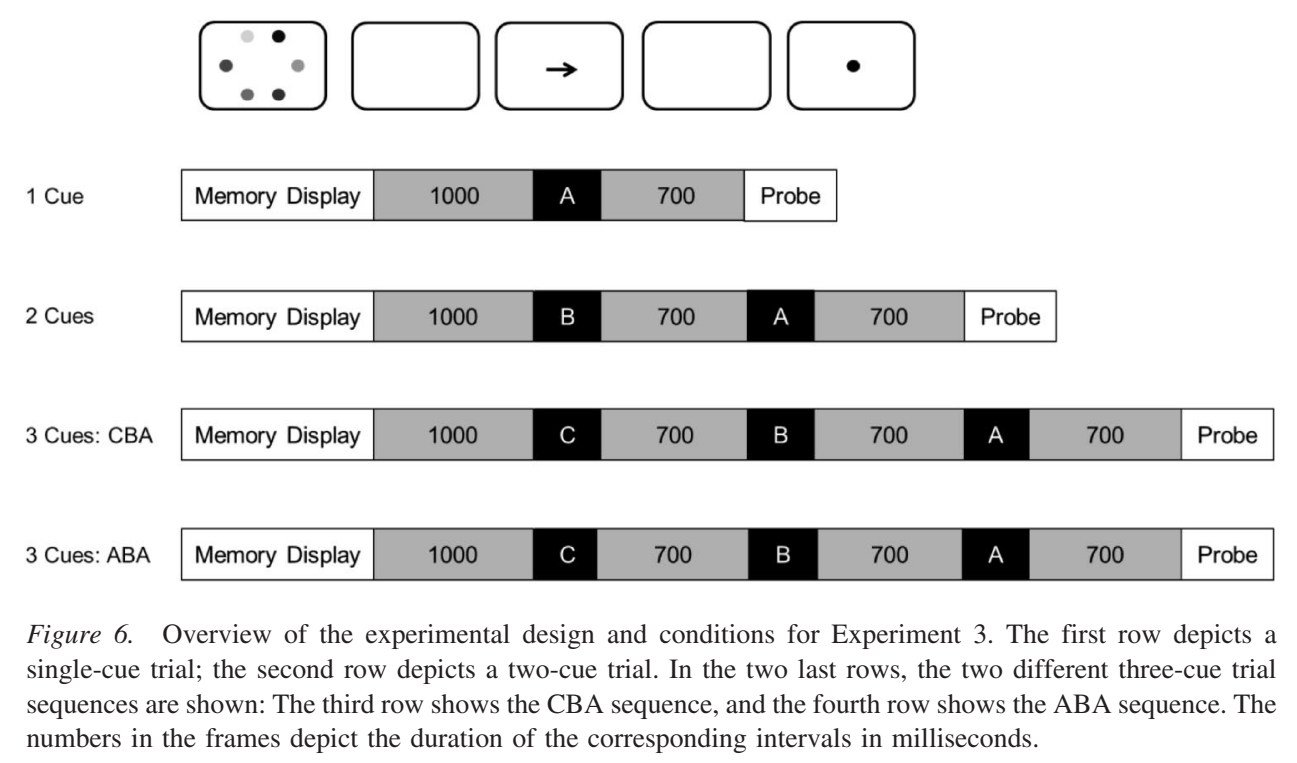


Table 4

Overview of the Number of Trials Per Probe Type in Experiment 3

\begin{tabular}{lcclc}
\hline & \multicolumn{3}{c}{ Probe type } \\
\cline { 2 - 5 } Cue condition & Match & New & Noncued intrusion & Previously cued intrusion \\
\hline Single cue & 120 & 40 & 40 (non), 40 (neigh) & - \\
Two cues & 120 & 30 & 30 (non), 30 (neigh) & 30 \\
Three cues & & & & 48 \\
CBA & 192 & 48 & 96 & 12 \\
ABA & 48 & 12 & 24 & \\
\hline
\end{tabular}

Note. Noncued intrusions $=$ probes matching an object that was not cued in the corresponding memory display; non = non-neighbors (probes matching an object that was not a neighbor of the last cued object); neigh $=$ neighbors (probes matching a neighbor of the last cued object).

(the second cue was selected at random from all items except A). The number of trials per probe condition can be found in Table 4.

Procedure. Each participant completed 24 practice trials prior to each of the two test sessions. Each session lasted about one hour and included 480 test trials, split into 10 blocks (the first nine comprising 50 and the last comprising 30 trials). The different cue conditions and probe types were presented in random order. Participants were instructed to answer as fast as possible, without committing errors.

\section{Results}

Percentage correct as well as RT data served as dependent variables, and data trimming and transformation was conducted as for Experiment 2. The overall average percentage correct was $78.8 \%(S D=7.1)$.

Number of cues. Our first analysis investigated whether information in WM can be maintained across up to three successive cues. A repeated-measures ANOVA on overall percentage correct for the three cue conditions (single-cue: $M=80.1 \%, S D=7.9$; two-cue: $M=77.9 \%, S D=8.0$; and three-cue: $M=78.6 \%, S D=$ 7.2) was marginally nonsignificant, $F(2,50)=2.963, p=.061$, partial $\eta^{2}=.106$. Pairwise comparisons revealed significantly better performance for single-cue than for two-cue trials ( $p=$ .015). The comparisons of single-cue trials to three-cue trials, as well as the comparison of two-cue trials to three-cue trials, yielded nonsignificant outcomes ( $p=.161$ and $p=.421$, respectively). The same repeated-measures ANOVA on log-transformed RT was significant, $F(2,50)=66.384, p<.001$, partial $\eta^{2}=.726$. Pairwise comparisons revealed significant differences for each single comparison (all $p$ values $<.001$ ), showing that single-cue trials (untransformed $M=.869 \mathrm{~s}, S D=.223$ ) led to the longest RTs and three-cue trials (untransformed $M=.727 \mathrm{~s}, S D=.175$ ) led to the shortest RTs, with two-cue trials (untransformed $M=$ $.819 \mathrm{~s}, S D=.208$ ) ranging between them. The opposite trends on accuracy and response speed suggest that, with increasing number of cues, participants tended to trade accuracy for speed. Together, there was little evidence in the present results of memory loss over a larger number of successive cues. Apparently, people have little difficulty shifting their focus of attention successively to up to three different items in WM.

Three-cue trial sequences. To explore the main question of whether the previously cued item is strengthened in WM or whether it is inhibited, we conducted a paired $t$ test for repeated measures, comparing the two three-cue trial sequences (CBA and ABA) to each other. The analysis for percentage correct revealed better performance for the ABA sequence $(M=82.5 \%, S D=7.2)$ than for the CBA sequence $(M=77.7 \%, S D=7.4), t(25)=$ $6.075, p<.001$. The same analysis on log-transformed RTs confirmed the pattern, with faster RTs for the ABA sequence (untransformed $M=0.698 \mathrm{~s}, S D=0.176$ ) than for the CBA sequence (untransformed $M=0.734 \mathrm{~s}, S D=0.175$ ), $t(25)=$ $-7.239, p<.001$. Both results unambiguously support the conclusion that the previously cued item is strengthened, rather than inhibited, upon leaving the focus of attention.

Furthermore, the ABA sequence produced significantly better performance than did two-cue trials, $t(25)=4.844, p<.001$, and single-cue trials, $t(25)=2.988, p=.006$, and significantly faster RTs, $t(25)=10.281, p<.001$ and $t(25)=13.255, p<.001$, respectively. The CBA sequence led to accuracy indistinguishable from that of two-cue trials, $t(25)=0.273, p=.787$, whereas RTs in the CBA trials were even faster than in the two-cue trials, $t(25)=6.255, p<.001$.

This experiment also offers another opportunity to look at intrusion effects from previously cued item probes (previously cued item refers to the first cued item in two-cue trials and to the second cued item in three-cue trials).

Mismatch probe type pattern. Analyses on log-transformed RT and accuracy data across mismatch probe conditions were conducted separately for each cue condition (single-cue, two-cue, and three-cue trials). The results of the planned contrasts on mismatch probe type (new, neighbor intrusion, non-neighbor intrusion, and previously cued intrusion probes) revealed the same overall pattern for each of the cue conditions, replicating the findings from Experiment 2. Results for each cue condition (single-cue, two-cue, and the CBA sequences) were computed separately. The ABA condition provided only a few observations per cell (see Table 4); therefore, analysis of ABA data was omitted.

Accuracy on new probes did not differ from accuracy on all other mismatch probes combined. Previously cued probes led to significantly higher accuracy than did noncued probes combined, replicating the key finding of Experiment 2. In single-cue and two-cue trials, neighbor probes resulted in worse performance than did non-neighbor probes (for the CBA sequence we did not distinguish neighbor and non-neighbor probes because of the sparseness of data). With regard to RTs, new item probes were responded 
Table 5

Planned Contrasts on Accuracy Data for the Different Mismatch Trial Conditions in Experiment 3

\begin{tabular}{lccc}
\hline \multicolumn{1}{c}{ Contrast } & $F(1,25)$ & partial $\eta^{2}$ & $p$ \\
\hline Single-cue trials & & & \\
$\quad$ New vs. [non-neighbors and neighbors] & 0.881 & .034 & .357 \\
$\quad$ Non-neighbors vs. neighbors & 4.423 & .150 & .046 \\
Two-cue trials & & & .421 \\
$\quad$ New vs. [previous and non-neighbors and neighbors] & 0.669 & .026 & .027 \\
$\quad$ Previous vs. [non-neighbors and neighbors] & 5.499 & .180 & .003 \\
$\quad$ Non-neighbors vs. neighbors & 11.001 & .306 & .761 \\
Three-cue trials & & & .004 \\
$\quad$ New vs. [previous and noncued] & 0.094 & .509 & $<.001$ \\
$\quad$ Previous vs. noncued & 25.940 & & \\
\hline
\end{tabular}

Note. Planned contrasts are shown for single-, two-, and three-cue trials (CBA sequences) for percentage correct.

to faster than all intrusion probes combined, and previously cued item probes did not differ from the noncued probes combined. Furthermore, non-neighbors and neighbors did not differ significantly from each other in single-cue and two-cue trials. The test statistics can be found in Tables 5 and 6, and Figure 5 provides a graphical overview of the results. Averages and the corresponding standard deviations for each condition (RT and accuracy) can be found in the Appendix.

\section{Discussion}

The comparison of the two three-cue trial sequences revealed significantly better performance and faster RTs for the ABA sequence than for the CBA sequence. This finding rules out the hypothesis that previously focused items are inhibited when another item is focused. If the previously focused item was inhibited, a shift back to that item in response to the final cue would have resulted in worse, not better, performance. The results of Experiment 3 further corroborate the conclusion that previously focused items remain strengthened, including strengthened item-to-context bindings. When these items are not cued as relevant by the final cue, their increased accessibility enables efficient recall to reject, as in Experiment 2. When these items are cued again as relevant by the final cue, as in the ABA sequences of the present experiment, their increased accessibility enables acceptance of matching probes with high speed and accuracy.
Our result is in line with an analogous observation in the multiple-probe study of Woodman and Vecera (2011). They probed participants on features of different objects in varying orders of objects: In their ABAC sequence, the third object tested was the same as the first; hence, this sequence requires a backshift similar to the one in our ABA sequence. In their ABCA sequence, the third object is different from all previously tested objects, corresponding to our CBA sequence. When performance on the third probe is compared between those two sequences, performance in the $\mathrm{ABAC}$ sequence was considerably better than performance for in the ABCA sequence. This matches the pattern we obtained by comparing the $\mathrm{CBA}$ to the $\mathrm{ABA}$ sequence, thereby supporting our finding of strengthened bindings due to focusing.

Replicating the findings from Experiment 2, previously cued probes showed equally slow RTs as did noncued intrusion probes. Hence, all intrusion probes from the memory display-including previously cued ones - resulted in significantly slower RTs than did new item probes. This pattern is in agreement with the dual-process theory of familiarity and recollection in WM (Oberauer, 2008), which postulates fast rejection of new item probes, on the basis of their relatively weak familiarity signal, and a slower recollection process to discriminate between probe types generating a stronger familiarity signal, such as intrusion probes and matching probes. The relatively slow response times for previously cued probes, in combination with the high accuracy of rejecting them, further bolsters the assumption

Table 6

Planned Contrasts on Log-Transformed RT Data for Mismatch Trial Conditions in Experiment 3

\begin{tabular}{lccc}
\hline \multicolumn{1}{c}{ Contrast } & $F(1,25)$ & partial $\eta^{2}$ & $p$ \\
\hline Single-cue trials log-RT & & & \\
New vs. [non-neighbors and neighbors] & 14.639 & .369 & .001 \\
Non-neighbors vs. neighbors & 0.009 & .000 & .927 \\
Two-cue trials log-RT & & & \\
New vs. [previous and non-neighbors and neighbors] & 5.530 & .181 & .027 \\
Previous vs. [non-neighbors and neighbors] & 0.108 & .004 & .745 \\
$\quad$ Non-neighbors vs. neighbors & 0.006 & .000 & .938 \\
Three-cue trials log-RT & & & \\
New vs. [previous and non-cued] & 21.445 & .462 & $<.001$ \\
Previous vs. non-cued & 0.208 & .008 & .652 \\
\hline
\end{tabular}

Note. Planned contrasts for single-, two-, and three-cue trials (CBA sequences) for log-transformed reaction time (RT) data. 
that the fate of defocused information in WM can be explained by the refreshing hypothesis: Temporarily focusing on an item strengthens its bindings to its location without reducing its activation relative to that of nonfocused items, so that it remains as familiar as noncued items but is easier to recollect.

Finally, we replicated the finding from Experiment 2 that neighbor probes were most difficult to reject. This finding further supports the notion of spatially imprecise representations in WM.

\section{General Discussion}

The present study investigated the fate of nonfocused information and the fate of previously focused and then defocused information in WM.

\section{The Fate of Nonfocused Information in WM}

The first two experiments (1A and $1 \mathrm{~B})$ revealed consistently that focusing one item in WM does not impair memory for the remaining nonfocused items. When onset of the valid cue as well as post-cue time were kept constant, single-cue and two-cue trials were responded to with equal accuracy.

Our pattern of results is difficult to accommodate by the discrete-capacity and constant-resource theories. Both theories predict that focusing on one representation in WM compromises the quality or accessibility of nonfocused information. In the resource model, a constant resource quantity is shared among items in the central component of WM. This implies that giving more of the resource to a single representation - the one prioritized by the first cue-draws some resource away from the remaining nonfocused items. For two-cue trials this implies that when the second cue appears, it points to a representation with reduced resource, such that this item must be harder to retrieve than is the cued item in single-cue trials. As a consequence, refocusing should lead to worse performance in two-cue trials than in single-cue trials. The same prediction derives from the discrete-capacity theory, which assumes that retro-cue benefits are obtained by assigning more than one slot to the cued representation. Accordingly, focusing one item comes at the expense of one nonfocused item, which must give up its slot. As a consequence, in two-cue trials the first cue increases the chance that the item cued second is no longer available because it has lost its slot to the first-cued item. This implies that, on average, accuracy should be worse on two-cue trials than on single-cue trials.

In contrast to this prediction, no difference was obtained between single-cue and two-cue trials. This result was predicted by the three-embedded-components model (Oberauer, 2002, 2009): Due to being in the focus of attention, the cued representation is particularly accessible, while the other representations in the direct-access region remain unaffected.

A comparison between a predictable condition, which could have discarded all noncued items upon seeing the first cue, and an unpredictable condition, which could not, failed to reveal a difference in accuracy in single-cue trials in Experiments 1A and 1B. This result indicates that both conditions maintained the entire memory display in WM, even though it would have been advantageous for the predictable condition to remove the noncued items. This finding is in contrast to results with the modified Sternberg task, in which people can remove one of two lists within one second after that list was declared irrelevant (Lewis-Peacock et al., 2012; Oberauer, 2001, 2005). One potentially relevant difference between studies in which removal effects were obtained and those in which no removal was observed is the structure of WM contents: Whereas in the modified Sternberg task, memory items are encoded as two separate sets, one of which is to be removed later, in the present retro-cue paradigm, one randomly selected item is cued as relevant, implying that the remaining items are irrelevant. It might be difficult for participants to remove a set of irrelevant items that is only formed ad hoc after encoding. Therefore, although the time we provided for removal might have been long enough to remove a predefined set, as suggested by evidence from several studies (Oberauer, 2001, 2002, 2005), this time might not have been sufficient for constructing an ad hoc set of irrelevant items and removing it.

\section{The Fate of Defocused Information in WM}

The second question we addressed concerns the fate of defocused information in WM. Experiments 2 and 3 showed that focusing an item strengthens its bindings to its context, and this gain in strength remains when the item is defocused later. Thereby, the defocused item can be recollected with high accuracy, resulting in a relatively high correct rejection rate. The advantage for the ABA sequence over the CBA sequence in Experiment 3 further supports the assumption that defocused items remain strengthened in memory and rules out the hypothesis that they are inhibited.

None of the three theoretical frameworks considered in the introduction make explicit predictions for the fate of defocused information, and none of them seem to readily be capable of explaining our evidence for strengthening of defocused information without further assumptions. We next discuss the results in light of the three theories and propose assumptions that could make them account for strengthening of defocused items.

The discrete-capacity theory can explain focusing by assuming that the focused item receives two slots. Extending this idea, we could assume that the defocused item retains its two slots even after the second item was cued. However, this would seriously reduce the accessibility of the nonfocused information. After two successive cues, four slots would be used, two for each of the two cued items. Assuming that the average young adult has four slots available (Cowan, 2005; Zhang \& Luck, 2008), no slot would be left, on average, for the remaining noncued items. Accordingly, we should expect performance hardly better than chance in the CBA sequence, in which three different items are cued, in contrast to much better performance in the two-cue (BA) sequence, in which only two items are cued. In fact, performance hardly differed between these two conditions.

The same argument applies to the resource model, although this model is more flexible because the resource can be divided up among items in continuous quantities. Assigning more of a constant resource to one representation comes at the expense of other representations' share of the resource. Strengthening of defocused items could be explained within this model by assuming that the defocused item retains some of its extra resource after the next item was cued. This would imply that the more items are cued in succession, the smaller the amount of resource left for the remaining items, including the one cued last and probed. By assuming that only a small quantity of extra resource remains with defocused 
items, the resource model might be able to accommodate our results. Because the resource cost for focusing a cued item is shared among all remaining items, each of them loses relatively little. As a consequence, the predicted loss in accuracy might be small. Quantitative modeling of a well-specified resource model of the retro-cue effect will be needed to decide whether such a model is compatible with our results.

A more serious problem for the resource theory arises from the contrast between probes matching a defocused item and probes matching a neighbor of the target item. Probes matching a defocused item were rejected better than the other intrusion probes, whereas probes matching a neighbor of the target were rejected worse than all other mismatch probe types. Within the constantresource model, the neighborhood effect can be explained by assuming that neighbors of the target item receive some of the extra resource assigned to the target item, perhaps by a spatially imprecise allocation of the resource. As a consequence, probes matching a neighbor are more familiar than probes matching nonneighbors. This assumption could explain the increased falsealarm rate to neighbors compared to nonneighbors. By the same assumption, the extra share of resources still residing with defocused items should lead to an increased false-alarm rate to probes matching a defocused item. We found the opposite. Assigning a larger share of resource to an item cannot be assumed to lead to more false alarms in the case of neighboring items and to fewer false alarms in the case of defocused items. Therefore, a resource theory cannot at the same time explain the neighborhood effect by assuming that resources spill over from the focused item to its neighbors, and explain strengthening of the defocused item by assuming that the defocused item retains some of its extra resource.

According to the three-embedded-components framework (Oberauer, 2002, 2009) items are held in the region of direct access by virtue of being bound to their contexts, and focused information is held in a separate focus of attention. The special status of the item selected as the content of the focus of attention explains the retro-cuing benefit. The theory so far makes no assumptions about defocused items. By default, defocused information would not be assumed to differ from other items in the direct-access region. To account for our finding of strengthening of defocused items, the model has to be augmented by the assumption that focusing an item strengthens its binding to its context in the direct-access region, and these bindings remain stronger than those of nonfocused items when the focus moves away. Note that this new assumption about defocused items is independent of what happens to the set of currently not focused items as a whole (i.e., nonfocused and defocused items). That set is maintained as long as needed, and apparently for some time even when no longer needed (as suggested by Experiments 1A and 1B), but will eventually be forgotten or removed. Whatever happens to the set of items in the direct-access region outside the focus, as long as there is some trace of them in the central component of WM, defocused items have stronger bindings to their contexts than nonfocused items.

An alternative explanation suggested to us for the reduced false-alarm rate for previously cued item probes is that the focus of attention expands to grasp both the previously cued and the lastcued item. This explanation is unattractive because in that case, performance should be impaired due to difficulties keeping apart the two items (which are highly confusable) in the focus of attention. If both the last-cued and the previously cued information were held in the focus, people would make more false alarms to probes matching the previously cued item, similar to the high false-alarm rate to neighboring probes (more on which below). We are forced to conclude that defocused information is maintained by different means than by staying in the focus of attention.

\section{Representations in WM Are Spatially Imprecise}

In addition to providing the answers to the main research questions outlined above, our experiments showed that representations in WM are spatially imprecise. Consistently, probes matching items spatially adjacent to the cued item (i.e., neighbor probes) led to worse accuracy than did the other intrusion probes. This pattern could be explained by spatially nondiscrete representations in WM: Objects bound to spatially neighboring locations are less distinct in memory than objects in more distant locations. The notion of imprecise, overlapping spatial representations in WM for simultaneous multi-object displays is analogous to the wellestablished notion of overlapping temporal positions in WM for sequentially presented items (Brown, Preece, \& Hulme, 2000; Burgess \& Hitch, 1999; Lewandowsky \& Farrell, 2008).

When an object cued by its location is retrieved into the focus of attention, the lack of spatial distinctiveness implies that not only the cued object itself but also some information from the neighboring objects enters the focus. When a neighboring item probe is then presented, it matches the partial information from this neighbor in the focus, resulting in a misleading match that causes the frequent false alarms to neighboring item probes. One implication of this view is that the focus of attention is not exclusively narrowed down on a single item; rather, the content of the focus may consist of a blend of several items. This idea matches well with our assumption that the focus of attention is not structurally limited. Rather, the focus of attention is a mechanism for selecting individual items in central WM (Oberauer \& Hein, 2012). It is limited to a single item to the extent that this selection is successful. The more items are held in the direct-access region at the same time and the more the contexts they are bound to overlap, the more difficult it is for the focus to narrow down on a single item at the exclusion of others.

\section{Conclusion}

In sum, the results of all experiments converge on the conclusion that WM representations that have never been focused after encoding remain accessible in the region of direct access. Focusing an item strengthens the bindings to its context, and these bindings remain strengthened relative to other items in the region of direct access after the item has been defocused. The maintenance of nonfocused information is predicted by the three-embeddedcomponents model (Oberauer, 2009) but not by two alternative theoretical frameworks, discrete-capacity theories and constantresource theories. The observation of strengthened item-context binding of defocused information was not predicted by either model. It can be accommodated by the three-embeddedcomponents model with the additional assumption that focusing refreshes item-context bindings in the direct-access region. The discrete-capacity and constant-resource theories face difficulties accommodating this result. 


\section{References}

Bao, M., Li, Z. H., Chen, X. C., \& Zhang, D. R. (2006). Backward inhibition in a task of switching attention within verbal working memory. Brain Research Bulletin, 69, 214-221. doi:10.1016/j.brainresbull .2005 .12 .007

Bays, P. M., Gorgoraptis, N., Wee, N., Marshall, L., \& Husain, M. (2011). Temporal dynamics of encoding, storage, and reallocation of visual working memory. Journal of Vision, 11, Article 6. doi:10.1167/11.10.6

Bays, P. M., \& Husain, M. (2008, August 8). Dynamic shifts of limited working memory resources in human vision. Science, 321, 851-854. doi:10.1126/science.1158023

Brainard, D. H. (1997). The Psychophysics Toolbox. Spatial Vision, 10, 433-436. doi:10.1163/156856897X00357

Brown, G. D. A., Preece, T., \& Hulme, C. (2000). Oscillator-based memory for serial order. Psychological Review, 107, 127-181. doi:10.1037/ 0033-295X.107.1.127

Burgess, N., \& Hitch, G. J. (1999). Memory for serial order: A network model of the phonological loop and its timing. Psychological Review, 106, 551-581. doi:10.1037/0033-295X.106.3.551

Chun, M. M., Golomb, J. D., \& Turk-Browne, N. B. (2011). A taxonomy of external and internal attention. Annual Review of Psychology, 62, 73-101. doi:10.1146/annurev.psych.093008.100427

Cowan, N. (1988). Evolving conceptions of memory storage, selective attention, and their mutual constraints within the human information processing system. Psychological Bulletin, 104, 163-191. doi:10.1037/ 0033-2909.104.2.163

Cowan, N. (1995). Attention and memory: An integrated framework. New York, NY: Oxford University Press.

Cowan, N. (1999). An embedded-process model of working memory. In A. Miyake \& P. Shah (Eds.), Models of working memory: Mechanisms of active maintenance and executive control (pp. 62-101). New York, NY: Cambridge University Press.

Cowan, N. (2001). The magical number 4 in short-term memory: A reconsideration of mental storage capacity. Behavioral and Brain Sciences, 24, 87-114. doi:10.1017/S0140525X01003922

Cowan, N. (2005). Working memory capacity. New York, NY: Psychological Press.

Cowan, N., Rouder, J. N., Blume, C. L., \& Saults, J. S. (2012). Models of verbal working memory capacity: What does it take to make them work? Psychological Review, 119, 480-499. doi:10.1037/a0027791

Cowan, N., Saults, J. S., Elliott, E. M., \& Moreno, M. V. (2002). Deconfounding serial recall. Journal of Memory and Language, 46, 153-177. doi:10.1006/jmla.2001.2805

Farrell, S., \& Lewandowsky, S. (2002). An endogenous distributed model of ordering in serial recall. Psychonomic Bulletin \& Review, 9, 59-79. doi:10.3758/BF03196257

Garavan, H. (1998). Serial attention within working memory. Memory \& Cognition, 26, 263-276. doi:10.3758/BF03201138

Glover, S., \& Dixon, P. (2004). Likelihood ratios: A simple and flexible statistic for empirical psychologists. Psychonomic Bulletin \& Review, 11, 791-806. doi:10.3758/BF03196706

Göthe, K., \& Oberauer, K. (2008). The integration of familiarity and recollection information in short-term recognition: Modeling speedaccuracy trade-off functions. Psychological Research, 72, 289-303. doi:10.1007/s00426-007-0111-9

Griffin, I. C., \& Nobre, A. C. (2003). Orienting attention to locations in internal representations. Journal of Cognitive Neuroscience, 15, 11761194. doi:10.1162/089892903322598139

Henson, R. N. A. (1998). Item repetition in short-term memory: Ranschburg repeated. Journal of Experimental Psychology: Learning, Memory, and Cognition, 24, 1162-1181. doi:10.1037/0278-7393.24.5.1162

Hübner, R., \& Druey, M. D. (2006). Response execution, selection, or activation: What is sufficient for response-related repetition effects under task shifting? Psychological Research, 70, 245-261. doi:10.1007/ s00426-005-0219-8

Johnson, M. K. (1992). MEM: Mechanisms of recollection. Journal of Cognitive Neuroscience, 4, 268-280. doi:10.1162/jocn.1992.4.3.268

Johnson, M. K., \& Hirst, W. (1993). MEM: Memory subsystems as processes. In A. F. Collins, S. E. Gathercole, M. A. Conway, \& P. E. Morris (Eds.), Theories of memory (pp. 241-286). Hove, England: Erlbaum.

Johnson, M. K., Raye, C. L., Mitchell, K. J., Greene, E. J., Cunningham, W. A., \& Sanislow, C. A. (2005). Using fMRI to investigate a component process of reflection: Prefrontal correlates of refreshing a justactivated representation. Cognitive, Affective \& Behavioral Neuroscience, 5, 339-361. doi:10.3758/CABN.5.3.339

Jolicoeur, P., \& Dell'Acqua, R. (1998). The demonstration of short-term consolidation. Cognitive Psychology, 36, 138-202. doi:10.1006/cogp .1998 .0684

Just, M. A., \& Carpenter, P. A. (1992). A capacity theory of comprehension: Individual difference in working memory. Psychological Review, 99, 122-149. doi:10.1037/0033-295X.99.1.122

Klein, R. M. (2000). Inhibition of return. Trends in Cognitive Sciences, 4 138-147. doi:10.1016/S1364-6613(00)01452-2

Koch, I., Gade, M., Philipp, A. M., \& Schuch, S. (2010). The role of inhibition in task switching: A review. Psychonomic Bulletin \& Review, 17, 1-14. doi:10.3758/PBR.17.1.1

Landman, R., Spekreijse, H., \& Lamme, A. F. (2003). Large capacity storage of integrated objects before change blindness. Vision Research, 43, 149-164. doi:10.1016/S0042-6989(02)00402-9

Lepsien, J., Griffin, I. C., Devlin, J. T., \& Nobre, A. C. (2005). Directing spatial attention in mental representations: Interactions between attentional orienting and working memory load. Neurolmage, 26, 733-743. doi:10.1016/j.neuroimage.2005.02.026

Lewandowsky, S., \& Farrell, S. (2008). Short-term memory: New data and a model. In B. H. Ross (Ed.), Advances in research and theory: Vol. 49 The psychology of learning and motivation (pp. 1-48). London, England: Elsevier.

Lewis-Peacock, J. A., Drysdale, A. T., Oberauer, K., \& Postle, B. R. (2012). Neural evidence for a distinction between short-term memory and the focus of attention. Journal of Cognitive Neuroscience, 24, 61-79. doi:10.1162/jocn_a_00140

Lin, P.-H., \& Luck, S. J. (2012). Proactive interference does not meaningfully distort visual working memory capacity estimates in the canonical change detection task. Frontiers in Psychology, 3, 1-9. doi:10.3389/ fpsyg.2012.00042

Luck, S. J., \& Vogel, E. K. (1997, November 20). The capacity of visual working memory for features and conjunctions. Nature, 390, 279-281. doi: $10.1038 / 36846$

Magnussen, S., \& Greenlee, M. W. (1999). The psychophysics of perceptual memory. Psychological Research, 62, 81-92. doi:10.1007/ s004260050043

Makovski, T., \& Jiang, Y. V. (2007). Distributing versus focusing attention in visual short term memory. Psychonomic Bulletin \& Review, 14, 1072-1078. doi:10.3758/BF03193093

Makovski, T., \& Jiang, Y. V. (2008). Proactive interference from items previously stored in visual working memory. Memory \& Cognition, 36, 43-52. doi:10.3758/MC.36.1.43

Makovski, T., Sussman, R., \& Jiang, Y. V. (2008). Orienting attention in visual working memory reduces interference from memory probes. Journal of Experimental Psychology: Learning, Memory, and Cognition, 34, 369-380. doi:10.1037/0278-7393.34.2.369

Masson, M. E. J. (2011). A tutorial on a practical Bayesian alternative to null-hypothesis significance testing. Behavior Research Methods, 43, 679-690. doi:10.3758/s13428-010-0049-5 
Matsukura, M., Luck, S. J., \& Vecera, S. P. (2007). Attention effects during visual short-term memory maintenance: Protection or prioritization? Perception \& Psychophysics, 69, 1422-1434. doi:10.3758/BF03192957

Maxcey-Richard, A. M., \& Hollingworth, A. (2012). The strategic retention of task-relevant objects in visual working memory. Journal of Experimental Psychology: Learning, Memory, and Cognition. Advance online publication. doi:10.1037/a0029496

Mayr, U., \& Keele, S. W. (2000). Changing internal constraints on action: The role of backward inhibition. Journal of Experimental Psychology: General, 129, 4-26. doi:10.1037/0096-3445.129.1.4

McElree, B. (2001). Working memory and focal attention. Journal of Experimental Psychology: Learning, Memory, and Cognition, 27, 817835. doi:10.1037/0278-7393.27.3.817

McElree, B., \& Dosher, B. A. (1989). Serial position and set size in short-term memory: The time course of recognition. Journal of Experimental Psychology: General, 118, 346-373. doi:10.1037/0096-3445 .118.4.346

Monsell, S. (1978). Recency, immediate recognition memory, and reaction time. Cognitive Psychology, 10, 465-501. doi:10.1016/00100285(78)90008-7

Morey, C. C., \& Bieler, M. (2012). Visual short-term memory always requires general attention. Psychonomic Bulletin \& Review. Advance online publication. doi:10.3758/s13423-012-0313-Z

Morey, C. C., \& Cowan, N. (2004). When visual and verbal memories compete: Evidence of cross-domain limits in working memory. Psychonomic Bulletin \& Review, 11, 296-301. doi:10.3758/BF03196573

Nairne, J. S. (1990). A feature model of immediate memory. Memory \& Cognition, 18, 251-269. doi:10.3758/BF03213879

Nobre, A. C., Griffin, I. C., \& Rao, A. (2008). Spatial attention can bias search in visual short term memory. Frontiers in Human Neuroscience, 1, Article 4. doi:10.3389/neuro.09.004.2007

Oberauer, K. (2001). Removing irrelevant information from working memory: A cognitive aging study with the modified Sternberg task. Journal of Experimental Psychology: Learning, Memory, and Cognition, 27, 948-957. doi:10.1037/0278-7393.27.4.948

Oberauer, K. (2002). Access to information in working memory: Exploring the focus of attention. Journal of Experimental Psychology: Learning, Memory, and Cognition, 28, 411-421. doi:10.1037/0278-7393.28.3.411

Oberauer, K. (2003). Understanding serial position curves in short-term recognition and recall. Journal of Memory and Language, 49, 469-483. doi:10.1016/S0749-596X(03)00080-9

Oberauer, K. (2005). Control of the contents of working memory-a comparison of two paradigms and two age groups. Journal of Experimental Psychology: Learning, Memory, and Cognition, 31, 714-728. doi:10.1037/0278-7393.31.4.714

Oberauer, K. (2006). Is the focus of attention in working memory expanded through practice? Journal of Experimental Psychology: Learning, Memory, and Cognition, 32, 197-214. doi:10.1037/0278-7393.32.2.197

Oberauer, K. (2008). How to say no: Single- and dual-process theories of short-term recognition tested on negative probes. Journal of Experimental Psychology: Learning, Memory, and Cognition, 34, 439-459. doi: 10.1037/0278-7393.34.3.439

Oberauer, K. (2009). Design for a working memory. Psychology of Learning and Motivation, 51, 45-100. doi:10.1016/S0079-7421(09)51002-X

Oberauer, K., \& Bialkova, S. (2009). Accessing information in working memory: Can the focus of attention grasp two elements at the same time? Journal of Experimental Psychology: General, 138, 64-87. doi: $10.1037 / \mathrm{a} 0014738$
Oberauer, K., \& Bialkova, S. (2011). Serial and parallel processes in working memory after practice. Journal of Experimental Psychology: Human Perception and Performance, 37, 606-614. doi:10.1037/ a0020986

Oberauer, K., \& Hein, L. (2012). Attention to information in working memory. Current Directions in Psychological Science, 21, 164-169. doi:10.1177/0963721412444727

Oberauer, K., \& Kliegl, R. (2004). Simultaneous cognitive operations in working memory after dual-task practice. Journal of Experimental Psychology: Human Perception and Performance, 30, 689-707. doi: 10.1037/0096-1523.30.4.689

Oberauer, K., \& Lange, E. (2009). Activation and binding in verbal working memory: A dual-process model for the recognition of nonwords. Cognitive Psychology, 58, 102-136. doi:10.1016/j.cogpsych .2008.05.003

Oberauer, K., \& Lewandowsky, S. (2008). Forgetting in immediate serial recall: Decay, temporal distinctiveness, or interference? Psychological Review, 115, 544-576. doi:10.1037/0033-295X.115.3.544

Oberauer, K., Lewandowsky, S., Farrell, S., Jarrold, C., \& Greaves, M. (2012). Modeling working memory: An interference model of complex span. Psychonomic Bulletin \& Review, 19, 779-819. doi:10.3758/ s13423-012-0272-4

Page, M. P. A., \& Norris, D. (1998). The primacy model: A new model of immediate serial recall. Psychological Review, 105, 761-781. doi: 10.1037/0033-295X.105.4.761-781

Pelli, D. G. (1997). The VideoToolbox software for visual psychophysics: Transforming numbers into movies, Spatial Vision, 10, 437-442. doi: $10.1163 / 156856897$ X00366

Pratt, J., Kingstone, A., \& Khoe, W. (1997). Inhibition of return in location- and identity-based choice decision tasks. Perception \& Psychophysics, 59, 964-971. doi:10.3758/BF03205511

Raye, C. L., Johnson, M. K., Mitchell, K. J., Greene, E. J., \& Johnson, M. R. (2007). Refreshing: A minimal executive function. Cortex, 43, 135-145. doi:10.1016/S0010-9452(08)70451-9

Rotello, C. M., \& Heit, E. (1999). Two-process models of recognition memory: Evidence for recall-to-reject? Journal of Memory and Language, 40, 432-453. doi:10.1006/jmla.1998.2623

Schmidt, B. K., Vogel, E. K., Woodman, G. F., \& Luck, S. J. (2002). Voluntary and automatic attentional control of visual working memory. Perception \& Psychophysics, 64, 754-763. doi:10.3758/BF03194742

Shipstead, Z., \& Engle, R. W. (2012). Interference within the focus of attention: Working memory tasks reflect more than temporary maintenance. Journal of Experimental Psychology: Learning, Memory, and Cognition. Advance online publication. doi:10.1037/a0028467

Sligte, I. G., Scholte, H. S., \& Lamme, V. A. F. (2008). Are there multiple visual short term memory stores? PLOS ONE, 3, e1699. doi:10.1371/ journal.pone.0001699

Woodman, G. F., \& Vecera, M. (2011). The cost of accessing an object's feature stored in visual working memory. Visual Cognition, 19, 1-12. doi:10.1080/13506285.2010.521140

Yonelinas, A. P. (2002). The nature of recollection and familiarity: A review of 30 years of research. Journal of Memory and Language, 46, 441-517. doi:10.1006/jmla.2002.2864

Zhang, W., \& Luck, S. J. (2008, May 8). Discrete fixed-resolution representations in visual working memory. Nature, 453, 233-235. doi 10.1038 /nature 06860

Zhang, W., \& Luck, S. J. (2009). Sudden death and gradual decay in visual working memory. Psychological Science, 20, 423-428. doi:10.1111/j 1467-9280.2009.02322.x 


\section{Appendix}

\section{Reaction Time and Accuracy Data for Experiments 2 and 3}

Table A1

RT Data per Probe Type and Cue Condition in Experiment 2

\begin{tabular}{|c|c|c|c|c|}
\hline \multirow[b]{2}{*}{ Cue condition } & \multicolumn{4}{|c|}{ Probe type } \\
\hline & Match & New & Noncued & Defocused \\
\hline Single cue short & $.569(.146)$ & $603(.139)$ & non: .627 (.178); neigh: .631 (.183) & - \\
\hline Single cue long & $.546(.139)$ & $.599(.148)$ & non: .618 (.151); neigh: .597 (.139) & - \\
\hline Two cues short & $.661(.191)$ & $.712(.177)$ & non: .773 (.285); neigh: .759 (.287) & $.747(.223)$ \\
\hline Two cues long & $.586(.186)$ & $.625(.183)$ & non: .658 (.187); neigh: .672 (.167) & $.633(.166)$ \\
\hline
\end{tabular}

Table A2

Accuracy Data per Probe Type and Cue Condition in Experiment 2

\begin{tabular}{|c|c|c|c|c|}
\hline \multirow[b]{2}{*}{ Cue condition } & \multicolumn{4}{|c|}{ Probe type } \\
\hline & Match & New & Noncued & Defocused \\
\hline Single cue short & $79.7(10.2)$ & $89.8(5.8)$ & non: 91.3 (7.6); neigh: 85.6 (10.8) & - \\
\hline Single cue long & $78.8(10.6)$ & $90.6(5.7)$ & non: 90.7 (8.8); neigh: 87.9 (6.8) & - \\
\hline Two cues short & $74.7(10.3)$ & $85.6(7.8)$ & non: 87.2 (12.7); neigh: 79.3 (11.9) & $88.3(8.1)$ \\
\hline Two cues long & $74.8(11.6)$ & $86.8(5.9)$ & non: 84.0 (11.4); neigh: 82.9 (12.9) & $90.0(8.4)$ \\
\hline
\end{tabular}

Table A3

RT Data per Probe Type and Cue Condition in Experiment 3

\begin{tabular}{|c|c|c|c|c|}
\hline \multirow[b]{2}{*}{ Cue condition } & \multicolumn{4}{|c|}{ Probe type } \\
\hline & Match & New & Noncued & Defocused \\
\hline Single cue & $.835(.238)$ & $.874(.216)$ & non: .905 (.225); neigh: .920 (.242) & - \\
\hline Two cues & $.780(.210)$ & $.825(.215)$ & non: $.863(.217)$; neigh: $.858(.214)$ & $.865(.258)$ \\
\hline CBA & $.696(.176)$ & $.735(.170)$ & $.784(.199)$ & $.779(.165)$ \\
\hline ABA & $.647(.176)$ & $.732(.172)$ & $.733(.187)$ & $.773(.237)$ \\
\hline
\end{tabular}

Note. Reaction time (RT) data are given in seconds (nontransformed), trimmed as described in the Method section. Standard deviations are provided in parentheses. Non-cued $=$ intrusion probes that were not cued in the corresponding memory display; Defocused $=$ defocused intrusion probe; non $=$ non-neighbor probes; neigh $=$ neighbor probes . 
Table A4

Accuracy Data per Probe Type and Cue Condition in Experiment 3

\begin{tabular}{lllcl}
\hline & \multicolumn{3}{c}{ Probe type } \\
\cline { 2 - 4 } Cue condition & Match & New & Noncued & Defocused \\
\hline Single cue & $75.6(11.7)$ & $85.4(9.9)$ & non: 85.6 (9.7); neigh: $82.5(7.9)$ & - \\
Two cues & $72.5(10.3)$ & $82.1(12.0)$ & non: $85.6(10.8) ;$ neigh: $79.5(11.3)$ & $86.0(11.2)$ \\
CBA & $74.4(9.7)$ & $81.5(9.3)$ & $78.2(8.7)$ & $85.7(8.8)$ \\
ABA & $78.8(9.4)$ & $87.2(12.1)$ & $85.7(11.8)$ & $85.9(11.7)$ \\
\hline
\end{tabular}

Note. Standard deviations are given in parentheses. Noncued $=$ intrusion probes that were not cued in the corresponding memory display; Defocused $=$ defocused intrusion probe; non $=$ non-neighbor probes; neigh $=$ neighbor probes.

Received February 28, 2012

Revision received November 2, 2012

Accepted November 5, 2012 